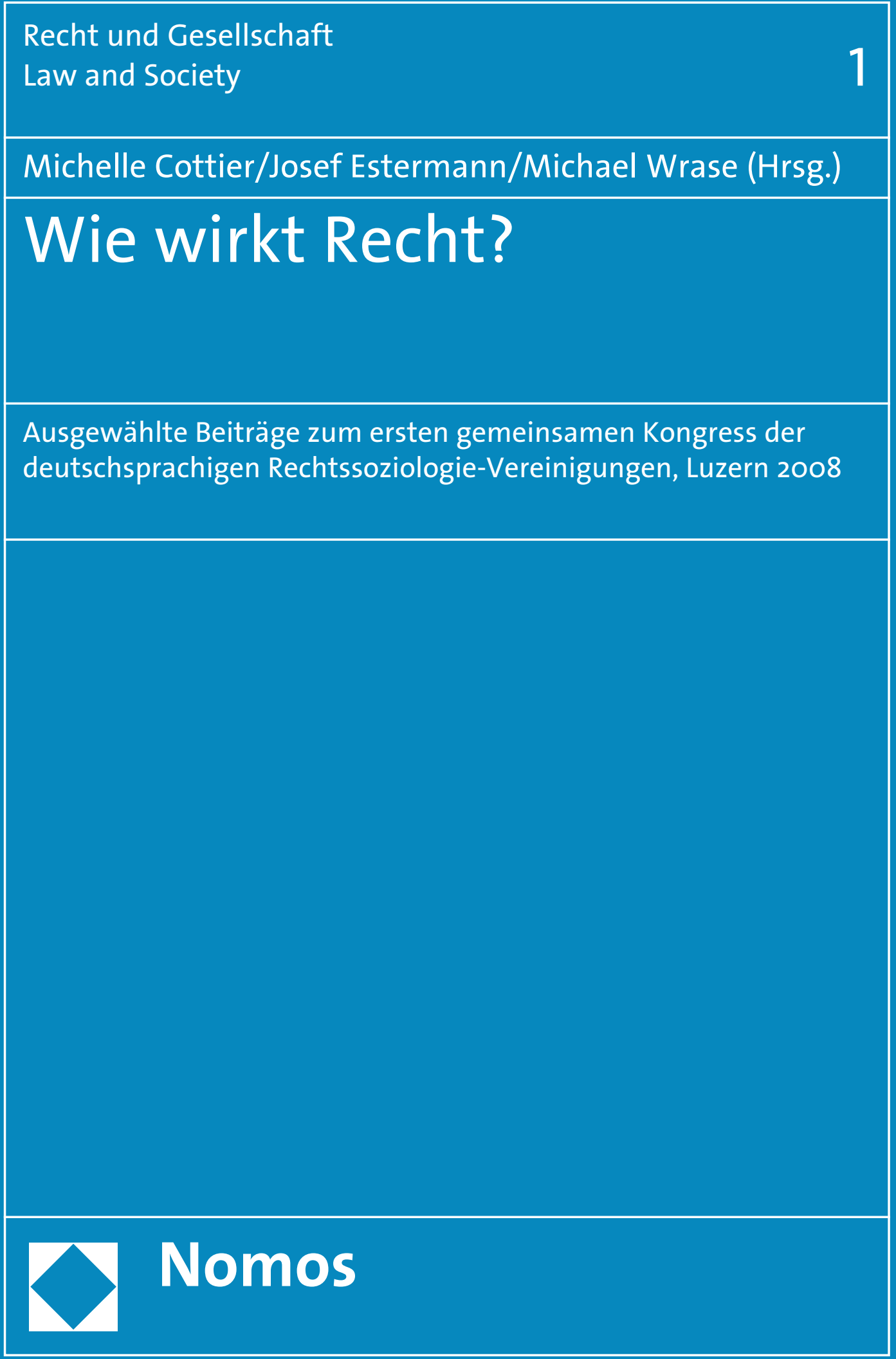





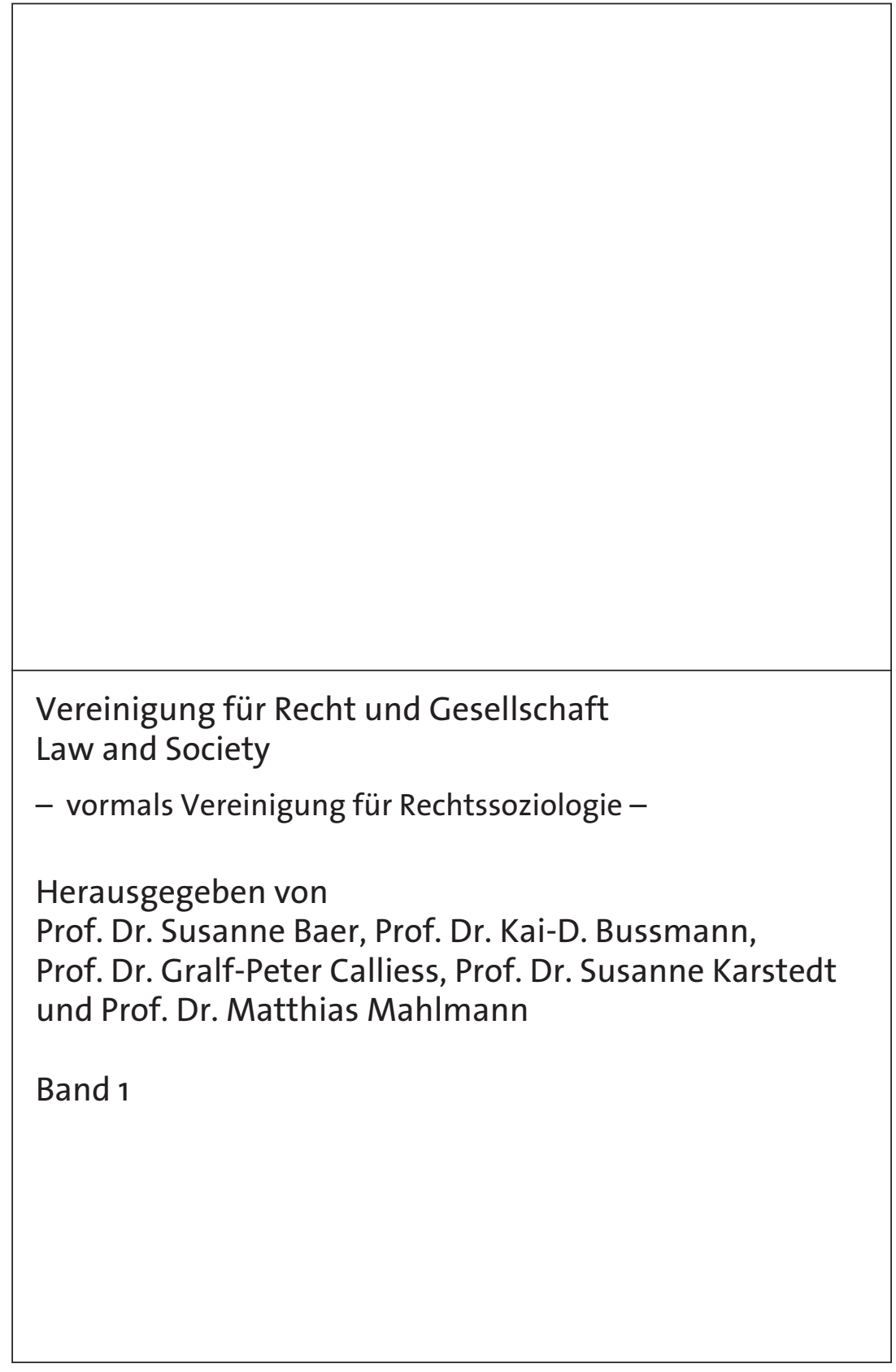


Michelle Cottier/Josef Estermann/Michael Wrase (Hrsg.)

\section{Wie wirkt Recht?}

Ausgewählte Beiträge zum ersten gemeinsamen Kongress der deutschsprachigen Rechtssoziologie-Vereinigungen, Luzern 4. - 6. September 2008

\section{Nomos}


Gedruckt mit Unterstützung des Bundesministeriums der Justiz, Berlin.

Die Deutsche Nationalbibliothek verzeichnet diese Publikation in der Deutschen Nationalbibliografie; detaillierte bibliografische Daten sind im Internet über http://dnb.d-nb.de abrufbar.

ISBN 978-3-8329-5397-3

\author{
1. Auflage 2010 \\ (c) Nomos Verlagsgesellschaft, Baden-Baden 2010. Printed in Germany. Alle Rechte, \\ auch die des Nachdrucks von Auszügen, der fotomechanischen Wiedergabe und der \\ Übersetzung, vorbehalten. Gedruckt auf alterungsbeständigem Papier.
}





\section{Inhaltsverzeichnis}

Michelle Cottier, Josef Estermann und Michael Wrase

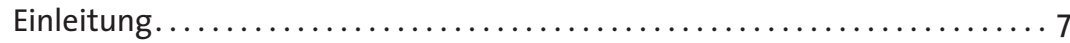

Hubert Rottleuthner und Margret Rottleuthner-Lutter

Recht und Kausalität ...

Inga Markovits

Rechtssoziologie für Außenseiter.

Erhard Blankenburg

Europäische Justizindikatoren: Budgets der Justiz, Richter und

Rechtsanwälte

Klaus F. Röhl

Crossover Parsifal

Josef Estermann

Die Verbindung von Recht und Soziologie als Chimäre

101

Michael Wrase

Recht und soziale Praxis - Überlegungen für eine soziologische

Rechtstheorie

Doris Mathilde Lucke

Was weiß Recht? Anmerkungen aus der sozialwissenschaftlichen Verwendungsforschung . .

Susanne Baer

Juristische Biopolitik: Das Wissensproblem im Recht am Beispiel „des“ demografischen Wandels.

Michelle Cottier

Soziologisches Wissen in Debatten um die Reformbedürftigkeit des Erbrechts. 


\section{Susanne Krasmann}

Die Folter im Recht

Dominique Grisard

Terrorismus, Schutz und Geschlecht. Entführung und Geiselnahme im schweizerischen Strafrecht. . .

Klaus F. Röhl

Die Macht der Symbole

Jens Newig

Symbolische Gesetzgebung zwischen Machtausübung und

gesellschaftlicher Selbsttäuschung. 301

Jochen Dreher

Zur Wirkungsweise von Kollektivsymbolik im Recht -

Symbolische Macht und „Klassenjustiz“

Alexander Klose

Wie wirkt Antidiskriminierungsrecht?

Barbara Willenbacher

Die Umgestaltung des Geschlechterkontraktes durch das

nacheheliche Unterhaltsrecht

Birte Hellmig

Recht als Verantwortungsinstanz - Ein empirischer Beitrag zu den

Funktionen von Recht

Alexandra Ortmann

Vom ,Motiv' zum ,Zweck'. Das Recht im täglichen Wandel -

das Beispiel der Reichsstrafprozessordnung 1879 ...

Lorenz Kähler

Zur Durchsetzungskraft abdingbaren Rechts. . 


\section{Einleitung}

\section{Michelle Cottier, Josef Estermann und Michael Wrase}

\section{Der Kongress „Wie wirkt Recht“}

„Wie wirkt Recht?“ Mit dieser Frage haben die deutschsprachigen Rechtssoziologie-Vereinigungen ${ }^{1}$ erstmals zu einem gemeinsamen Kongress geladen, der vom 4. bis 6. September 2008 in Luzern stattfand. Dabei richtete sich der Call for Papers nicht nur an die Rechtssoziologie, sondern sprach auch benachbarte Disziplinen an, die sich unter der Bezeichnung „Recht und Gesellschaft“ oder „interdisziplinäre Rechtsforschung“ zusammenfassen lassen. Der Einladung folgten daher nicht nur Vertreterinnen und Vertreter der Rechtssoziologie im engeren Sinne, sondern auch der Rechtsethnologie, Rechtsphilosophie, Literaturwissenschaft, Rechtsgeschichte, Politikwissenschaft, Theologie und Psychologie. Diese disziplinäre Öffnung wie auch der große Erfolg des Kongresses, der mit ungefähr 160 Vorträgen und 250 Teilnehmenden als der größte in der Geschichte der deutschsprachigen Rechtssoziologie bezeichnet werden kann, bestätigten die Vermutung, welche die Programmkommission in der Einladung zur Abschlussdiskussion des Kongresses aufgestellt hatte: „Die deutschsprachige interdisziplinäre Rechtsforschung befindet sich zur Zeit im Umbruch. Es beginnt sich ein größerer Forschungszusammenhang, Recht und Gesellschaft ${ }^{\star}$ abzuzeichnen, der über die Grenzen der klassischen Rechtssoziologie hinausgeht und die aktive $\mathrm{Zu}$ sammenarbeit aller mit Recht befassten Disziplinen stärker sucht als bisher.“ Es ist zu hoffen, dass es der Nachfolgeveranstaltung, die im September 2011 unter dem Titel „Der Kampf ums Recht“ (Jhering 1872) in Wien stattfinden soll (vgl. auch Estermann 2009), gelingen wird, den Elan des Luzerner Kongresses aufzunehmen und den Forschungszusammenhang „Recht und Gesellschaft" weiter zu etablieren.

Eine Auswahl der während des Luzerner Kongresses präsentierten Beiträge wurde von der Programmkommission zur Veröffentlichung ausgewählt. Mit dem vorliegenden Band ist die Reihe der in allen drei beteiligten Län-

1 Beteiligt an der Organisation des Kongresses waren das Forschungskomitee Rechtssoziologie und Rechtswirklichkeitsforschung der Schweizerischen Gesellschaft für Soziologie, die Sektion Rechtssoziologie der Deutschen Gesellschaft für Soziologie, die Vereinigung für Rechtssoziologie, der Berliner Arbeitskreis Rechtswirklichkeit und das Institut für Rechts- und Kriminalsoziologie Wien. 
dern erschienenen Publikationen nun komplett. Weitere Beiträge sind 2009 in der Zeitschrift für Rechtssoziologie (Damm 2009, Höland 2009, Struck 2009), im Band „Citizen by Proxy und Individualrechte“ (Kreissl 2009) und im Band „Interdisziplinäre Rechtsforschung zwischen Rechtswirklichkeit, Rechtsanalyse und Rechtsgestaltung“ (Estermann 2009) erschienen.

\section{Zu den Beiträgen in diesem Band}

Die Autorinnen und Autoren der 19 Beiträge im vorliegenden Band ließen sich von der Frage „Wie wirkt Recht?" herausfordern, provozieren, inspirieren und bieten eine Fülle von theoretisch und empirisch fundierten Antworten an. Einige Beiträge nehmen zudem Stellung zum Nutzen eines um das Begriffspaar „Recht und Gesellschaft“ gruppierten wissenschaftlichen Zusammenhangs.

Der erste Beitrag von Hubert Rottleuthner und Margret Rottleuthner-Lutter bezieht sich direkt auf das Kongressthema und nimmt es zum Anlass, sich unter dem Titel „Recht und Kausalität“ mit den methodischen Problemen der Erforschung von Rechtswirkungen zu befassen. Sie weisen ganz im Sinne des didaktischen Klassikers „Störche und Geburtenrate“ auf die auch hier bestehende Gefahr des vorschnellen Schlusses von der Korrelation auf die Kausalität hin. Anhand der Diskussion aktueller empirischer Studien unter anderem zu den deutschen Gesetzen zum Kündigungsschutz, zum Beschäftigtenschutz (Schutz vor sexueller Belästigung), zur Prostitution und zum Elterngeld weisen die Autorin und der Autor darauf hin, dass insbesondere die Kontrolle von Drittvariablen eine methodische Absicherung gewährleisten kann. Schließlich werfen sie die Frage nach einer spezifisch „sozialen Kausalität“ in Abgrenzung zu psychologischen Wirkungszusammenhängen auf.

Die Beiträge von Inga Markovits, Erhard Blankenburg und Klaus F. Röhl wurden im Rahmen der von Michael Wrase moderierten Session „Das Recht zwischen den Disziplinen - Rechtsforschung als Bewegung? “ präsentiert. Die Beiträge beleuchten aus unterschiedlicher, teilweise auch kritischer Perspektive wesentliche Themen, Methoden und Entwicklungen der Rechtssoziologie im breiteren Kontext der Law \& Society-Forschung.

Inga Markovits „Rechtssoziologie für Außenseiter“ ist ein Plädoyer für eine von einem strengen methodischen Korsett befreite, auf der Immersion der Forscherin in ihr Forschungsfeld und dem Dialog zwischen Forscherin und ihren Daten beruhenden Forschungsprozess. In ihrem Werkstattbericht über die Entstehung des Buches Gerechtigkeit in Lüritz, das die 40jährige Tätigkeit eines DDR-Amtsgerichts beschreibt, zeigt sie auf, wie anhand des 
Gerichtsalltags an einem erstinstanzlichen Gericht Geist und Charakter einer ganzen Rechtsordnung wie auch deren Wandel fassbar werden.

Der Beitrag von Erhard Blankenburg nimmt die Makroperspektive eines Rechtsvergleichs ein. Er nähert sich der Rechtswirklichkeit der Justiz von der Seite ihrer Kosten her: In einem Vergleich von sechs westeuropäischen und fünf post-kommunistischen Mitgliedstaaten der EU erläutert er, wie Ausgaben für die Justiz als Indikator für Rechtsstaatlichkeit verwendet werden können. Enorme Unterschiede zwischen den Ländern zeigen sich etwa in Bezug auf die Gewährung von sozialer Rechtshilfe oder der Zahl der im professionellen Rechtsstab beschäftigten Personen. Anhand von Hintergrundinformationen zu den Justizsystemen der behandelten Länder liefert der Beitrag Interpretationen und Erklärungen für diese innereuropäischen Differenzen.

Klaus F. Röhl reagiert auf die Einladung zum Kongress, die auf das Vorbild der Law \& Society-Bewegung Bezug nahm, mit einem Essay unter dem Titel „Crossover Parsifal“. Ein musikalischer Crossover zwischen Bachs Matthäus-Passion und Wagners Parsifal verwendet er als Bild für gewisse Tendenzen in der Rechtssoziologie, sich in ein "Crossover" mit kulturwissenschaftlichen Ansätzen zu begeben. Die Rechtssoziologie riskiere damit, ihre disziplinären Konturen und ihre ungleichheits- und herrschaftskritische Stossrichtung zu verlieren.

Der im Band folgende Beitrag von Josef Estermann fokussiert die obligate Inter- und Transdisziplinarität der Rechtssoziologie. Er plädiert für eine kritische Betonung der Konstitutionsbedingungen von Soziologie als ontologische Wissenschaft in der Nachfolge von Durkheim und Weber und von der Rechtswissenschaft als stets normativ bezogene Wissenschaft. Dabei geht es weniger um die der Soziologie zugeschriebene Herrschaftskritik, sondern um die kritische Beschreibung des Faktischen, welche die Soziologie im Gegensatz zur normativen Rechtswissenschaft zu leisten in der Lage ist. Dabei dürfe sich die Rechtssoziologie nicht auf die Rolle der ancilla iuris einschränken lassen, sondern müsse eine Form annehmen, in welcher die (deontologischen und ontologischen) Glieder der Chimäre klar erkennbar bleiben, aber zu einem Wesen zusammengeführt sind. Das Ziel sei also nicht eine Auflösung der disziplinären Konturen von Soziologie und Rechtswissenschaft, sondern deren dialektische Schärfung.

Der Beitrag von Michael Wrase stellt, ausgehend vom soziologischen Individualismus Max Webers, die Frage nach einer soziologischen Rechtstheorie, die das Recht als soziale Praxis von Rechtsakteuren beschreibt und erklärt. Er stützt sich hierbei vor allem auf das Habitus-Feld-Konzept Pierre Bourdieus und dessen Arbeiten zum ,juristischen Feld‘. Im Vergleich 
mit der Systemtheorie Niklas Luhmanns arbeitet er Gemeinsamkeiten und Unterschiede der theoretischen Konzepte von ,sozialem Feld‘ und ,System heraus. Abschließend formuliert der Autor einige Gedanken, wie sich der sozialkritische Ansatz Bourdieus im Rahmen einer soziologisch fundierten Grundrechtstheorie fruchtbar machen lässt.

Die folgenden drei Beiträge sind in der Session „Wissensrezeption in Rechtskontexten“ präsentiert worden. Sie bewegen sich im Spannungsfeld dreier möglicher rechtssoziologischer Perspektiven: Es sind dies die Hoffnung auf besseres Recht durch „Soziologisierung“ der Rechtswissenschaft, eine distanziertere, wissen(schaft)ssoziologische Zugangsweise mit eher nüchterner Einschätzung der Möglichkeiten der Optimierung von Recht durch außerrechtliches Wissen und schließlich eine an Michel Foucault anschließende Analyse, die von einer engen Verbindung von Wissen und Macht ausgeht.

Der als Einleitung und Grundierung der Session angelegte wissenssoziologische Beitrag von Doris Mathilde Lucke wendet die den Kongress begleitende Frage „Wie wirkt Recht?“ zu „Was weiß Recht?“. Zunächst setzt sich Lucke mit der sozialwissenschaftlichen Verwendungsforschung auseinander, die es sich zur Aufgabe gemacht hat, den „Erfolg“ soziologischer Forschung an der Rezeption ihrer Erkenntnisse in Politik und Recht zu messen. Deren Grundannahme, dass das Recht durch mehr wissenschaftlich generiertes Wissen besser und gerechter werde, stellt sie grundlegend in Frage und schlägt als „Verwendungsforschung revisited“ eine ganz neue Perspektive vor, die den Wert von „Nichtwissen“ des Rechts betont, die Legitimitätshierarchien des vom Recht rezipierten Wissens kritisch reflektiert und sich mit dem durch zunehmende Entkoppelung gekennzeichneten Verhältnis von Alltagswissen und Recht befasst.

Susanne Baer analysiert in ihrem Beitrag das „Wissensproblem im Recht“ anhand der Bezugnahme des Rechts auf Debatten um den „demografischen Wandel“" und macht deutlich, wie das Recht demografische Daten und Bilder verwendet, um Biopolitik zu betreiben. Sie weist insbesondere auf die Bedeutsamkeit von (sozialen) Kategorisierungen als Grundlage für Diskriminierung und auf die mit dem Diskurs zum demografischen Wandel verbundenen normalisierenden Homogenitätsvorstellungen von „Volk“ hin. Abschließend setzt sie sich mit einer Reihe von „Techniken im Umgang mit dem Wissensproblem“ auseinander und wirft die Frage auf, inwiefern auch die rechtliche Bezugnahme auf Erfahrungs- und Alltagswissen Teil haben kann am biopolitischen Projekt, z. B. indem ontologisierende Naturalisierungen unreflektiert verwendet werden. Insofern wird hier eine skeptischere Haltung gegenüber der Annäherung des Rechts an das Alltagswissen eingenommen als im Beitrag von Lucke. 
Der dritte Beitrag der Session von Michelle Cottier reiht sich schließlich in die Tradition der soziologischen Jurisprudenz ein und hat einen dementsprechend optimistischeren Zugang zu den Möglichkeiten der Wissensrezeption durch Recht. Thema des Beitrags sind innerjuristische Debatten um die Reformbedürftigkeit des Erbrechts angesichts der Pluralisierung der Familienformen. In der rechtswissenschaftlichen Vorstellung, dass das Erbrecht familiären „Normalverhältnissen“ und dem „mutmaßlichen Erblasserwillen“ folgen sollte, wie auch in der Verwendung des Topos der „intergenerationellen Solidarität“ erblickt die Autorin eine Tendenz zur Homogenisierung von Familie. Sie plädiert in der Folge für den Einbezug qualitativer Sozialforschung zur Vielfalt der Lebensrealitäten in Patchwork-Familien in die Reformdiskussionen und die Entwicklung neuer, an die veränderten Lebensrealitäten angepasster Regelungstechniken.

Ebenfalls mit rechtlichen Wissenspolitiken befasst sich Susanne Krasmann in ihrem Beitrag „Die Folter im Recht“. Die Praxis der Folter, wie sie heute auch in rechtsstaatlich-demokratischen Staaten praktiziert wird, versteht sie in einem Kontext der aktuellen Sicherheitsimperative. Die Terrorismusbekämpfung nach 9/11 findet gemäß der Autorin unter einer Logik der Vorbeugung statt, in der die konkrete Bedrohung unbekannt ist und zugleich eminent und nicht tolerabel, weshalb der Suche nach Anhaltspunkten keine Grenzen gesetzt sind. Die aktuelle Logik der Folter, die sich als Mittel ausschließlich zur Erlangung von Informationen (und nicht zur Erlangung eines rechtlich verwertbaren Geständnisses) versteht, ist laut Krasmann in analoger Weise von einem unbedingten Willen zum Wissen strukturiert, der die „Wahrheit" erzwingen will.

Dominique Grisard schließt thematisch insofern an, als auch hier - bezogen auf den Zeitraum der 1970er und 1980er Jahre - der sicherheitspolitische Kontext des Rechts thematisiert wird. Thema ist eine Revision des Schweizer Strafgesetzbuches von 1981, mit der - angesichts seinerzeit aktueller Geiselnahmen und Entführungen durch nahöstliche Befreiungsbewegungen und linksterroristische Gruppierungen - der vormals auf Frauen beschränkte Entführungstatbestand auch auf männliche Opfer ausgeweitet wurde. In einer am Ansatz Law as Narrative orientierten Analyse von parlamentarischen und rechtswissenschaftlichen Darstellungen arbeitet die Autorin heraus, wie rund um die Revision eine Reartikulation von Geschlecht und strafrechtlichem Schutz in Bezug auf den männlichen Staatsbürger stattgefunden hat.

Die nächsten drei Beiträge sind in der Session präsentiert worden, die sich der rechtssoziologischen Symboltheorie gewidmet hat. Die Ankündigung der Session drückt die Hoffnung aus, mit dem Symbolbegriff das um Normen 
herum kreisende „Universum von Bedeutungen“ einzufangen, „die nicht nur an Texte, sondern auch an Orte oder Gegenstände, bestimmte Wortfolgen, Handlungen oder Bilder anknüpfen“.

Der Beitrag von Klaus F. Röhl mit dem Titel „Die Macht der Symbole“ spricht sich für einen den Zwecken der Rechtssoziologie gemäßen engen Symbolbegriff aus. Er solle sich „auf Bedeutungen konzentrieren, die nicht als semantische Abbildung erfassbar sind, sondern auf eine unbestimmtere, eben ,symbolische' Weise vermittelt werden." Symbolisch in diesem engeren Sinne sei nur der „Nebensinn“ eines Bedeutungsträgers, der allerdings auch zur Hauptsache werden könne. Entsprechend sind gemäß Röhl Gesetze nur „symbolisch“, wenn und soweit der mit ihnen verbundene Nebensinn die Vorherrschaft gewinnt.

Newig dagegen definiert symbolische Gesetzgebung als Gesetzgebung, die sich durch niedrige antizipative rechtlich-sachliche Effektivität sowie eine hohe antizipative politisch-strategische Effektivität auszeichnet. Aus der Sicht eines „Public-Choice-Ansatzes“ symbolischer Gesetzgebung analysiert er zwei Fallbeispiele aus dem deutschen Umweltrecht. Gemäß Newigs Analyse wird symbolische Gesetzgebung begünstigt durch ein hohes Maß öffentlicher Aufmerksamkeit zu einem Thema, ein ungünstiges Kosten-NutzenVerhältnis einer sachlich-materiellen Regelung, intensive und wertgeladene Konflikte, Informationsasymmetrien zugunsten des Gesetzgebers sowie eine hohe Komplexität des Regelungsgegenstandes.

Im letzten Beitrag der symboltheoretischen Session mit dem Titel „Zur Wirkungsweise von Kollektivsymbolik im Recht“ fragt Jochen Dreher nach der sozialen Funktion von Symbolen, deren Wirkungsmacht er insbesondere in der Etablierung und Integration sozialer Entitäten sieht. Anhand eines Fallbeispiels, den gerichtlichen Verfahren zur „Volkswagen-Affäre“ des Jahres 2005, in der es um illegale Sonderzahlungen an Arbeitnehmervertreter ging, zeigt er auf, wie die öffentliche Wahrnehmung der justiziellen Verarbeitung der Affäre als „Klassenjustiz“ nur innerhalb einer auch durch das Recht mitgetragenen symbolischen Strukturierung des sozialen Raums und der symbolischen Konstitution von Machtdifferenzen zu verstehen ist.

Der Beitrag von Alexander Klose „Wie wirkt Antidiskriminierungsrecht?“ knüpft wiederum an das Generalthema des Luzerner Kongresses an. Zunächst diskutiert er zwei aktuelle Studien, die zu unterschiedlichen Ergebnissen bezüglich der Folgekosten des deutschen „Allgemeinen Gleichbehandlungsgesetzes“ (AGG) kommen, aus einer methodenkritischen Perspektive. Anschließend geht er mit Hilfe der rechtssoziologischen Theorien von Opp, Diekmann und Rottleuthner den möglichen Faktoren nach, die die Wirksamkeit des AGG beeinflussen. Positiv könnten gemäß dieser Analyse die 
im AGG vorgesehenen prozessualen Erleichterungen und die Pflichten der Arbeitgeber zur Information über das AGG wirken. Negativ ins Gewicht fällt hingegen die geringe gesellschaftliche Akzeptanz für verschiedene der im Gesetz normierten Diskriminierungsverbote, die nicht zuletzt durch die ungewöhnliche Schärfe der Kritik am Gesetzgebungsprojekt in Fachpresse und Medien verursacht wurde und die eine Situation der „normativen Abweichung “ begünstigte.

Eine weitere deutsche Gesetzesnovelle mit großen gesellschaftspolitischen Implikationen steht in Barbara Willenbachers Beitrag zur „Umgestaltung des Geschlechterkontraktes durch das nacheheliche Unterhaltsrecht “ im Zentrum. Sie analysiert die neueste Revision des Unterhaltsrechts, die geschiedene und nicht verheiratete Mütter in Bezug auf die Erwerbsobliegenheit gleichstellt und dem Kindesunterhalt höchste Priorität verleiht. Das Ziel der Reduktion der Kinderarmut wird jedoch gemäß der Autorin nicht erreicht werden können. Die Autorin prognostiziert anhand von soziologischen Erkenntnissen zur sozio-ökonomischen Situation alleinerziehender Mütter, dass die aufgrund der Revision gezwungermaßen zunehmende Erwerbstätigkeit alleinstehender Mütter nicht notwendig zu einer Erhöhung ihres Haushaltseinkommens führen wird.

Das richterliche Entscheiden ist Thema des Beitrags von Birte Hellmig mit dem Titel „Recht als Verantwortungsinstanz“. Ausgangspunkt ist die oftmals vorgetragene Diagnose eines Auseinanderklaffens von Theorie im Sinne von juristischer Methodenlehre und tatsächlich praktizierter gerichtlicher Rechtsanwendung. Der Beitrag stellt dieser Einschätzung anhand einer Auswertung von Interviews mit Arbeitsrichterinnen und -richtern die These entgegen, es existiere eine große Varianz von richterlichen Herangehensweisen: Erstens ein Typus, der „Recht als Störfaktor“ bei der sachgerechten Lösung zwischenmenschlicher Konflikte empfindet, zweitens ein Typus, der „Recht als Entscheidungsmaßstab“ wahrnimmt und eng am Gesetz arbeitet und drittens ein Typus, der „Recht als kommunikative Herausforderung“ und Rechtsprechen als Übersetzungsaufgabe definiert. Zentral ist aber laut der Autorin für alle Interviewten die grundsätzliche Frage, wie die Verantwortung für das Rechtsprechen verteilt ist.

Ebenfalls mit dem richterlichen Handeln und Auseinandersetzungen um die richtige Methode der Gesetzesauslegung befasst sich der rechtshistorische Beitrag von Alexandra Ortmann. Ihr Fallbeispiel ist die Umsetzung der neuen Reichsstrafprozessordnung von 1879, die insbesondere die Stellung des Angeklagten stärkte. Der Beitrag zeigt anhand einer Analyse der juristischen Fachliteratur, wie die Durchsetzung der teleologischen Auslegungsmethode, die am „objektiven Zweck“ einer Norm ausgerichtet ist, ermöglichte, 
dass trotz eines komplett revidierten Strafprozessrechts auch ältere Interpretationen der Ziele des Strafprozesses, etwa die Erzielung eines Geständnisses als Hauptziel der Vernehmung, in der Praxis zum neuen Recht erhalten blieben. Rechtswandel erscheint so als dynamischer Prozess des Durchmischens von alten und neuen Rechtsmeinungen. Die dadurch entstehenden breiten Interpretationsspielräume führten gemäß der Autorin zu einer großen ,juristischen Varianz'.

Der den Band abschließende Beitrag von Lorenz Kähler „Zur Durchsetzungskraft abdingbaren Rechts“ kehrt zur Frage der Wirkung von Recht zurück und schließt damit den Kreis. Er setzt sich mit dem Phänomen auseinander, dass sich - wie empirische Studien gezeigt haben - abdingbares Recht trotz der Möglichkeit seiner vertraglichen Abbedingung zumeist in der Praxis durchsetzt. Die gängigen Erklärungen etwa mit den Kosten der Abbedingung, den strategischen Nachteilen der Thematisierung von Risiken in Vertragsverhandlungen oder dem „omission bias“ (wonach ein negatives Ergebnis schlechter bewertet wird, wenn es auf eine eigene Handlung zurückgeht als auf eine Unterlassung) greifen gemäß dem Autor zu kurz. Er ergänzt sie durch den bisher noch unbeachtet gebliebenen Faktor des Vertrauens der Vertragsparteien zueinander und in die ihren Vertrag überformende Rechtsordnung.

\section{Dank}

Bei der Realisierung der vorliegenden Publikation haben wir vielfältige Unterstützung erfahren: Zunächst einmal danken wir den Herausgeberinnen und Herausgebern der neuen Reihe „Recht und Gesellschaft“ wie auch dem Nomos Verlag für die dem Tagungsband zukommende Ehre, als erster Band erscheinen zu können. Sodann danken wir den anonymen Gutachterinnen und Gutachtern, die uns mit ihrer Expertise aus den verschiedensten Fachbereichen bei der Auswahl der 19 Beiträge aus über 60 Einreichungen unterstützt haben. Reiner Koll und Paul Skidmore danken wir für das umsichtige Lektorat, Valentin Funk für den sorgfältigen Satz und das professionelle Layout. Schließlich sind wir für die großzügige finanzielle Unterstützung sehr dankbar: Eine Vielzahl von Institutionen aus der Schweiz, Deutschland und Österreich haben den Kongress „Wie wirkt Recht?“ mit ihren Beiträgen möglich gemacht. ${ }^{2}$ Das deutsche Bundesministerium der Justiz hat zudem die Druckkosten für diesen Band übernommen.

2 Der Kongress wurde unterstützt von dem Schweizerischen Nationalfonds zur Förderung der wissenschaftlichen Forschung (SNF), der Schweizerischen Akademie der 


\section{Literatur}

Damm, Reinhard (2009) Wie wirkt „Nichtrecht“? Genesis und Geltung privater Regeln am Beispiel medizinischer Professionsnormen, Zeitschrift für Rechtssoziologie 30: $3 \mathrm{ff}$.

Estermann, Josef (Hrsg.) (2009) Interdisziplinäre Rechtsforschung zwischen Rechtswirklichkeit, Rechtsanalyse und Rechtsgestaltung. Beiträge zum Kongress „Wie wirkt Recht?“"Luzern, 2008, Bern/Beckenried/Luzern.

Höland, Armin (2009) Wie wirkt Rechtsprechung?, Zeitschrift für Rechtssoziologie 30: $23 \mathrm{ff}$.

JHering, Rudolf von (1872) Der Kampf um’s Recht, Wien.

Kreissl, Reinhard (Hrsg.) (2009) Citizen by Proxy und Individualrechte. Über das Rechtssubjekt und seine Stellvertreter (= Bd. 3 Schriften zur Rechts- und Kriminalsoziologie, IRKS, Wien), Münster.

Struck, Gerhard (2009) Instrumentalisierung verschiedener Wirkungsformen von Recht in einem Vertragstext - eine Fallstudie, Zeitschrift für Rechtssoziologie 30: $47 \mathrm{ff}$.

Geistes- und Sozialwissenschaften (SAGW), der Schweizerischen Gesellschaft für Soziologie (SGS), der Forschungskommission der Universität Luzern, dem Schweizerischen Bundesamt für Justiz, Bern, dem Deutschen Bundesministerium der Justiz, Berlin, dem Österreichischen Bundesministerium für Justiz, Wien, und dem Österreichischen Bundesministerium für Wissenschaft und Forschung, Wien. 


\title{
Soziologisches Wissen in Debatten um die Reformbedürftigkeit des Erbrechts
}

\author{
Michelle Cottier*
}

\begin{abstract}
s
Angesichts der soziologischen Gegenwartsdiagnose einer Pluralisierung der Familienformen stellt sich für das Erbrecht die Frage, wie es sich an die veränderten Lebensrealitäten anpassen soll. In den innerjuristischen Debatten um die Reform des Erbrechts wird zwar die Notwendigkeit des Wandels „vom Status zur Realbeziehung“ erkannt, nach wie vor wird aber von einer homogenisierenden Sichtweise von "Familie“ ausgegangen. Die sozialwissenschaftliche Forschung zeigt dagegen eine große Heterogenität erbrechtlicher Verfügungen und normativer Erwartungen in den „neuen“ Familienformen auf. In der Perspektive gilt es, im Rabmen einer interdisziplinär abgestützten Erbrechtsreform sozialwissenschaftliches Wissen über das „lebende Erbrecht“ in die Entwicklung von neuen, der Vielfalt aktueller Familienrealitäten gerechter werdenden Regelungstechniken einfließen zu lassen.
\end{abstract}

\section{Sociological knowledge in debates on the need for succession law reform}

In the light of a sociological diagnosis of a pluralisation in family forms, succession law faces the issue of how to adapt to the changed social realities. In the internal legal debates on succession law reform, although the need for change "from status to relationship" is acknowledged, a homogenising concept of "the family" remains. In contrast, social science research reveals a considerable variety in the dispositions upon death and normative expectations among members of "new" family forms. From that perspective, therefore, a succession law reform based on interdisciplinary efforts is needed, which incorporates social science knowledge on "living succession law" in

\footnotetext{
* Dieser Beitrag ist im Rahmen des vom Schweizerischen Nationalfonds zur Förderung der wissenschaftlichen Forschung finanzierten Projekts „Das Erbrecht angesichts der Pluralisierung der Familienformen“ entstanden. Für wertvolle Anregungen danke ich Roland Fankhauser, Vaios Karavas und Michael Wrase.
} 
the development of new regulatory techniques which are better adjusted to the needs of the diversity of current family realities.

\section{Einleitung}

Nach jahrzehntelangem Schattendasein steht das Erbrecht aktuell wieder stärker im Fokus der Aufmerksamkeit. Zwar bildet das Erbrecht nicht in gleicher Weise Gegenstand breiter gesellschaftspolitischer Debatten wie noch im 19. Jahrhundert, ${ }^{1}$ es läuft zur Zeit jedoch im deutschsprachigen Raum eine immer größere Kreise ziehende innerjuristische Diskussion um die Reformbedürftigkeit dieses Rechtsgebiets. Für die rechtssoziologische Frage nach den Bedingungen und Grenzen der Wissensrezeption in Rechtskontexten ${ }^{2}$ bietet die Debatte spannendes Anschauungsmaterial, da sie sich unter anderem intensiv mit den gesellschaftlichen Voraussetzungen erbrechtlicher Regelungen befasst. ${ }^{3}$

Innerhalb der Reformdebatten zum materiellen Erbrecht können verschiedene Diskussionsstränge unterschieden werden, die ausdrücklich auf gesellschaftliche Veränderungsprozesse Bezug nehmen. So wird zunächst die These eines Funktionswandels des Erbrechts kontrovers diskutiert: Demnach habe das Erbrecht des 19. Jahrhunderts aufgrund der damals noch geringeren Lebenserwartung die Funktion gehabt, der nachfolgenden Generation den Eintritt in die wirtschaftliche Selbständigkeit zu erleichtern. Diese Funktion sei durch die gestiegene Lebenserwartung weggefallen, weshalb Pflichtteilsregelungen zugunsten der Nachkommen in Frage zu stellen seien. ${ }^{4}$ Eine weitere, wiederum umstrittene These geht vom Wandel der Groß- zur Kleinfamilie aus und behauptet, dass der Gesetzgeber beim Erlass des Erbrechts am Ende

1 Vgl. Beckert 2004; Willekens 2006.

2 So das Thema der gemeinsam mit Susanne Baer und Doris Lucke veranstalteten Session anlässlich der Konferenz „Wie wirkt Recht?“ vom 4. bis 6. September 2008 an der Universität Luzern.

3 Eigentliche inter- oder transdisziplinäre Bemühungen sind dagegen eher selten. Eine Ausnahme bildet das derzeit laufende Projekt „Generationen in der Erbengesellschaft Ein Deutungsmuster soziokulturellen Wandels“ unter der Leitung von Prof. Sigrid Weigel (Literaturwissenschaft), Prof. Martin Kohli (Soziologie) und Prof. Peter Breitschmid (Rechtswissenschaft), www.generationenforschung.de.

4 Vgl. Coing 1972: A 112 ff. Diese These stellt Otte wiederum mit Bezugnahme auf Daten der historischen Demographie in Frage, indem er aufzeigt, dass auch Ende des 19. Jahrhunderts die Nachkommen beim Tod ihrer Eltern meist schon im Alter der wirtschaftlichen Selbständigkeit waren; vgl. Otte 2002: 335 ff. 
des 19. Jahrhunderts am Bild der Großfamilie orientiert gewesen sei und in der Zwischenzeit aufgrund des Wandels hin zur Kleinfamilie die Legitimation für ein gesetzliches Erbrecht der entfernten Verwandten abhanden gekommen sei. ${ }^{5}$ In jüngster Zeit hat sich nun der Akzent auf die Frage der Anpassung des Erbrechts an die „Pluralisierung der Familienformen“, und der Notwendigkeit eines Wandels „vom Status zur Realbeziehung“ verschoben. ${ }^{6}$ Bei letzterer Diskussion setzt der vorliegende Beitrag an.

Die im Folgenden untersuchten Reformdebatten sind zwar eng mit politischen Gesetzgebungsprozessen verbunden, etwa im Rahmen von Fachtagungen mit rechtspolitischer Zielrichtung ${ }^{7}$ oder in der Mitwirkung juristischer Expertinnen und Experten bei der Ausarbeitung von Gesetzesentwürfen. Die Reformdiskussionen - jedenfalls im Bereich des materiellen Erbrechts spielen sich jedoch größtenteils innerhalb eines spezialisierten Fachdiskurses $\mathrm{ab}$ und sind kaum an eine größere Öffentlichkeit gerichtet. ${ }^{8}$

Der Untersuchungsgegenstand „Soziologisches Wissen in Debatten um die Reformbedürftigkeit des Erbrechts“ präsentiert sich unterschiedlich, je nachdem, ob die (wissens-) soziologische Frage nach der Verwendung soziologischen Wissens oder die rechtswissenschaftliche Frage nach dem Nutzen und der Methode des Einbezugs soziologischer Konzepte und Erkenntnisse in Überlegungen zur Rechtsentwicklung in den Vordergrund gerückt wird. Beide Perspektiven sollen im Folgenden eingenommen werden. Nach einem Überblick über den Inhalt der Reformdebatten werden Bedingungen und Grenzen der Rezeption sozialwissenschaftlichen Wissens untersucht. Dabei werden die rechtswissenschaftlichen Figuren der „Normalverhältnisse“ und des „durchschnittlichen Erblassers“ sowie der Topos der „intergenerationellen Solidarität“ einer genaueren Analyse unterzogen. Anschließend werden die Resultate aktueller empirischer Studien zum Erben in „neuen Familienformen“ zusammengefasst, um auf dieser Basis Perspektiven für die Entwicklung einer interdisziplinär abgestützten Erbrechtsreform entwerfen zu können. Der Beitrag reiht sich damit in die Tradition der „soziologischen Jurisprudenz “ ein. ${ }^{9}$ Diese stößt zwar in der Rechtssoziologie in jüngerer Zeit

5 Vgl. Leipold 1980: 173 ff.; kritisch Otte 2002: 340 ff., Linker 1999: 78 ff., Bauszus 2006: passim.

6 Vgl. Röthel in Lipp/Röthel/Windel 2008: 109 ff. mit Bezugnahme auf Schwenzer 1987; Breitschmid 2007a.

7 Vgl. Martiny 2002; Röthel 2007; Wolf 2007.

8 Eine größere Öffentlichkeitswirkung hat das mit der Frage der Umverteilung verknüpfte Thema der Erbschaftssteuern, vgl. etwa die auf Kissling 2008 und Seibt 2008 folgende Debatte in der Leserbriefspalte des Tagesanzeigers vom 16., 17. und 26. Mai 2008.

9 Vgl. Röhl 1987: 43 ff. 
auf immer größere Skepsis und der Glaube an ein „besseres Recht“ durch Integration von soziologischen Konzepten und empirischem Wissen wird zunehmend in Frage gestellt. ${ }^{10}$ Diese Bedenken sollen im Folgenden ernst genommen werden, ohne aber eine gegenüber dem interdisziplinären Austausch völlig pessimistisch eingestellte Perspektive einzunehmen.

\section{Das Erbrecht und die Pluralisierung der Familienformen}

\section{Statusorientierung des geltenden Erbrechts und veränderte gesellschaftliche Realitäten}

In Deutschland und der Schweiz orientiert sich heute das Erbrecht ausschließlich am familienrechtlichen Status: ${ }^{11}$ So werden in beiden Rechtsordnungen in der gesetzlichen Erbfolge und im Pflichtteilsrecht Personen berücksichtigt, die entweder über Verwandtschaft (Nachkommen, Eltern, weitere Verwandte) oder eine formalisierte Partnerschaft (Ehe, eingetragene Partnerschaft) mit dem so genannten Erblasser verbunden sind. Realbeziehungen ${ }^{12}$ wie insbesondere nichteheliche Partnerschaften und Stiefkind- und Pflegekindbeziehungen, finden in den beiden Ländern keine Berücksichtigung. ${ }^{13}$

Die Rechtswissenschaft kommt aber nicht um die soziologische Gegenwartsdiagnose der „Pluralisierung der Familienformen“14 herum. Jedenfalls im Vergleich mit dem „Golden Age of Marriage“ der fünfziger Jahre mit der auf lebenslanger Ehe beruhenden Kleinfamilie als Standardlebensform, ${ }^{15}$ zeigt sich eine größere Vielfalt an gelebten Familien- und Lebensformen: Die Zunahme von Ehescheidungen führt dazu, dass immer mehr Menschen im Laufe ihres Lebens verschiedene Partnerschaften eingehen und insbesonde-

10 So etwa Lucke 1988; Monahan/Walker 2008; Teubner 1990. Optimistischer nun aber Teubner 2004: 12.

11 Vgl. Breitschmid 2007a; Lipp/Röthel/Windel 2008.

12 Zum Wandel des Familienrechts vom Status zur Realbeziehung grundlegend Schwenzer 1987.

$13 \mathrm{Vgl}$. das Schweizer Bundesgericht: „Die gesetzliche Erbfolge stellt ausschliesslich auf formelle familienrechtliche Beziehungen $a b$ und legt innerhalb der Familie eine bestimmte Stufenfolge fest. Ob die Beziehung tatsächlich gelebt wurde, ist ebenso unerheblich, wie eine tatsächlich gelebte Beziehung (z.B. Konkubinat, Pflegeverhältnis u.ä.), die ohne formelle familienrechtliche Bande keine gesetzliche Erbberechtigung verschaffen kann.“ BGE 134 III 467 (Urteil vom 25.6.2008).

14 Vgl. etwa Peuckert 2008: $23 \mathrm{ff}$.

15 Die Familiensoziologie weist allerdings mit Blick auf die historische Familienforschung darauf hin, dass bereits vor der Industrialisierung eine große Variabilität der Familienformen existiert hat (Peuckert 2008: 17). 
re auch Kinder aus verschiedenen Beziehungen haben. In Folge dessen ist das Zusammenleben mit Kindern aus verschiedenen Partnerschaften (neu zusammengesetzte oder „Patchwork-Familien“), aber auch der Abbruch einer einmal eingegangenen Eltern-Kind-Beziehung, eine zunehmend gelebte Familienrealität. Zudem sind nichteheliche Lebensgemeinschaften mit oder ohne Kinder in Zunahme begriffen. Parallel zur Pluralisierung gelebter Familienarrangements hat sich ein Wandel auf der Ebene der familienbezogenen Werte und des Selbstverständnisses von Familien vollzogen, der zu einer Deinstitutionalisierung der bürgerlichen Familienform ${ }^{16}$ und der Akzeptanz einer Vielfalt von Varianten von Eltern- und Partnerschaft geführt hat. ${ }^{17}$ Dies hat sich nicht zuletzt in Reformen des Familienrechts niedergeschlagen, etwa in der Gleichstellung von nichtehelichen und ehelichen Kindern, der Schaffung von Partnerschaftsgesetzen für gleichgeschlechtliche Paare oder der rechtlichen Absicherung von nichtehelichen Lebensgemeinschaften. ${ }^{18}$

Das Erbrecht ist bis vor kurzem von den Anpassungsbemühungen des Rechts an pluralisierte Familienrealitäten weitgehend unberührt geblieben. ${ }^{19}$ Dies hat sich in jüngster Zeit verändert und es wird insbesondere die Erweiterung des Kreises der gesetzlichen Erben und die Abschaffung oder Flexibilisierung des Pflichtteilsrechts diskutiert. ${ }^{20}$

\section{Erweiterung des Kreises der gesetzlichen Erben}

Die Feststellung einer Pluralisierung der Familienformen und -werte führt zur Frage, ob der Kreis der gesetzlichen Erben, also der Personen, die Erbenstellung haben, wenn die Erblasserin oder der Erblasser keine Verfügungen über sein Vermögen getätigt hat, ${ }^{21}$ auch auf Realbeziehungen erweitert werden soll.

In historischer Perspektive haben sich in diesem Bereich in Europa seit der Schaffung der nationalen zivilrechtlichen Kodifikationen Ende des 19., Anfang des 20. Jahrhunderts die größten Änderungen vollzogen. Zu nennen ist insbesondere die Beseitigung der Diskriminierung nichtehelicher Kinder und deren Gleichstellung als gesetzliche Erben mit den ehelichen Kindern sowie die Verbesserung der Stellung des überlebenden Ehegatten. ${ }^{22}$

\footnotetext{
16 Tyrell 1988.

17 Lettke 2006.

18 Vgl. Schwenzer 2007: 708 ff.; Lettke 2007.

19 Vgl. Willekens 2006.

20 Vgl. auch die Diskussion um das Ehegattenerbrecht im Kontext von Trennung und Scheidung, insbesondere Fankhauser 2007.

21 Vgl. etwa BaslerKomm/Stähelin, Vorbemerkung Art. 457-466 ZGB.

22 Vgl. Willekens 2006: $517 \mathrm{ff}$.
} 
Gegenwärtig wird nun diskutiert, auch nichteheliche Lebenspartnerinnen und -partner in den Genuss eines gesetzlichen Erbrechts kommen zu lassen. Sowohl Gegnerinnen wie Befürworter stützen ihre Argumentation auf „soziale Tatsachen“: Für eine solche Erweiterung spreche, dass eine sehr große Zahl von Paaren unverheiratet zusammenlebten ${ }^{23}$ und dass die meisten nicht daran denken würden, mittels Testament oder Erbvertrag ihren Nachlass zu regeln, was zur Folge habe, dass die überlebende Partnerin leer ausgehe. ${ }^{24}$ Dagegen spreche, dass die meisten nichtehelichen Lebensgemeinschaften nur als „Probephase“ vor der Ehe gedacht seien und eben explizit keine Verrechtlichung ihrer Beziehung wünschten. ${ }^{25}$

Zum anderen wird über ein gesetzliches Erbrecht von Stiefkindern nachgedacht. Die Befürworter schlagen zum Beispiel einen neuen Status Stiefkindschaft vor, der durch einen formalen rechtsgeschäftlichen Akt begründet werden müsste und der sich von der Adoption dadurch abgrenzt, dass das Kindesverhältnis zum zweiten leiblichen Elternteil nicht aufgelöst wird. An diesen könnten dann die elterliche Sorge des Stiefelternteils und ein gesetzliches Erbrecht anknüpfen. ${ }^{26}$

\section{Abschaffung oder Flexibilisierung des Pflichtteilsrechts}

Die weitaus intensiver geführte Diskussion dreht sich um die Zukunft des Pflichtteilsrechts. Dieses sichert bestimmten Personen einen festen Anteil am Erbe und beschränkt damit die Testierfreiheit, die Freiheit also, über das eigene Vermögen über den Tod hinaus zu verfügen. Das schweizerische und das deutsche Recht sehen eine ähnliche, wenn auch nicht deckungsgleiche Pflichtteilsregelung vor:

In der Schweiz ist das Pflichtteilsrecht grundsätzlich als eigentliches „Noterbrecht“ (von „notwendige Erben“)27 ausgestaltet und bei einer Pflichtteilsverletzung kann mit der Herabsetzungsklage die Erbenstellung erlangt wer-

23 Strätz 2001: 457: „Bei dieser Größenordnung von mehreren Millionen nichtehelicher Paare halte ich es für lebensfremd, ihnen unter Hinweis auf die heute praktisch unbeschränkte Testiermöglichkeit ein gesetzliches Erbrecht vorzuenthalten."

24 Strätz 2001: 457 f.; Rauscher 1993: Bd II/2, 270 ff.; Breitschmid 2007a: 16f.; Strebel 2008: 1031 .

25 Röthel in Lipp/Röthel/Windel 2008: 111 ff.; Röthel 2006; Wolf 2007: $310 \mathrm{f}$.

26 Muscheler 2006: 198.

27 Vgl. Druey 2002: 57. 
den. ${ }^{28}$ In Deutschland ist der Pflichtteilsanspruch anders als im Schweizer Recht nur ein obligatorischer Anspruch gegenüber dem Nachlass. ${ }^{29}$

Die Höhe des Pflichtteils nach Schweizer Recht beträgt für Nachkommen drei Viertel des gesetzlichen Erbanspruchs. Für jeden Elternteil des Erblassers (bei Abwesenheit von Nachkommen), für den überlebenden Ehegatten und die eingetragene Partnerin oder den eingetragenen Partner beträgt er die Hälfte. ${ }^{30}$ In Deutschland steht der Pflichtteil den Nachkommen, bei Abwesenheit von Nachkommen den Eltern, dem überlebenden Ehegatten ${ }^{31}$ und dem eingetragenen Lebenspartner zu. ${ }^{32}$ Der Pflichtteil beträgt hier für alle Berechtigten die Hälfte des gesetzlichen Erbteils. ${ }^{33}$

In beiden Ländern hat die Erblasserin die Möglichkeit, den Pflichtteil zu entziehen oder zu beschränken; diese ist aber sehr restriktiv ausgestaltet. Im Wesentlichen ist eine solche Entziehung in beiden Ländern bei schweren Straftaten gegen den Erblasser oder ihm nahe verbundene Personen, bei Verletzung familienrechtlicher Pflichten (insbesondere Unterhaltspflichten) und bei Überschuldung möglich. ${ }^{34}$

Besonders in Deutschland ist die Literatur zur Frage der Reform des Pflichtteilsrechts mittlerweile kaum mehr überblickbar. ${ }^{35}$ Diskutiert wird eine Abschaffung oder zumindest Reduktion respektive Flexibilisierung des Pflichtteilsrechts insbesondere der Nachkommen. Die faktischen Konstellationen, in denen problematische Auswirkungen des geltenden Pflichtteilsrechts festgestellt werden, sind dabei heterogen: Im Vordergrund steht die Situation der Weitergabe des Familienunternehmens an die nächste Generation. Der Pflichtteil kann dort zu Schwierigkeiten führen, wo ein Nachkomme das Familienunternehmen übernehmen soll und eine Auszahlung der Pflichtteile

28 Hausheer/Aebi-Müller 2001: 219. Diese Folge kann der Erblasser oder die Erblasserin umgehen, indem er oder sie den Pflichtteilsberechtigten zwar von der Erbenstellung ausschließt, aber eine dem Wert des Pflichtteils entsprechende Zuwendung unter Lebenden tätigt, ein Vermächtnis hinterlässt oder mit einer Teilungsvorschrift bestimmte Gegenstände dem Pflichtteilsberechtigten zuweist, Art. 522 Abs. 1 ZGB, vgl. BaslerKomm/Forni/Piatti, Art. 522 ZGB.

29 Vgl. etwa Reimann 2001: 46.

30 Art. 457 ff., 471 ZGB.

31 \2303 BGB.

$32 \rrbracket 10 \mathrm{LPartG}$.

$33 \llbracket 2303$ Abs. 1 BGB.

34 \2333 ff. BGB; Art. 477 ff. ZGB. Zur Reform des deutschen Pflichtteilsentziehungsrechts sogleich unten.

35 Vgl. für Deutschland nur Coing 1972; Rauscher 1993; Schlüter 2000; Dauner-Lieb 2001; Otte 2002; Martiny 2002; Röthel (Hrsg) 2007; Lipp/Röthel/Windel 2008; Staudinger/Haas, BGB, Neubearbeitung 2006, Einl. zu $\$ \mathbb{S} 2303$ ff. Rn. 17 ff. m. w. Nachw. Für die Schweiz: Schaer 1976; Breitschmid 2001; Breitschmid 2007a; Eitel 2003; Wolf 2007. 
an die anderen Nachkommen zu Eingriffen in die Substanz des Unternehmens zwingt. ${ }^{36}$ Des Weiteren wird der Pflichtteilsschutz als Hindernis für gemeinnützige Zuwendungen betrachtet. ${ }^{37}$ Die im Kontext der „Pluralisierung der Familienformen“ besonders bedeutsame und aufgrund der hohen Scheidungsrate zunehmend anzutreffende Konstellation ist schließlich diejenige, dass eine Eltern-Kind-Beziehung nicht mehr gelebt wird, dass sich also Elternteil (in der Praxis zumeist der Vater) und Kind „entfremdet“ haben. Einige Stimmen in der Literatur fordern, dass im Sinne der österreichischen Regelung ${ }^{38}$ eine Reduktion des Pflichtteils oder ein gänzlicher Ausschluss bei „Zerrüttung“ des Eltern-Kind-Verhältnisses möglich sein soll. ${ }^{39}$

In Deutschland sind den Reformgelüsten allerdings verfassungsrechtliche Grenzen gesetzt: Das deutsche Bundesverfassungsgericht stellte in seinem Beschluss vom 19. April 2005 fest, dass das Grundgesetz (Art. 14 Abs. 1 Satz 1 in Verbindung mit Art. 6 Abs. 1 GG) eine grundsätzlich unentziehbare und bedarfsunabhängige wirtschaftliche Mindestbeteiligung der Kinder des Erblassers an dessen Nachlass gewährleiste. ${ }^{40}$ Die Entscheidung wird so interpretiert, dass das Grundgesetz einer Abschaffung des Pflichtteils entgegensteht, dass aber der Gesetzgeber einen gewissen Gestaltungsspielraum in Bezug auf dessen konkrete Ausgestaltung hat. ${ }^{41}$ Insbesondere die Einführung eines Zerrüttungstatbestandes wird aber als nicht verfassungskonform betrachtet. ${ }^{42}$

Entsprechend der verfassungsrechtlichen Grundentscheidung wird in der jüngsten Reform des Erbrechts in Deutschland ${ }^{43}$ denn auch nicht das Pflichtteilsrecht an sich in Frage gestellt. Die Revision beschränkt sich auf „punktuelle Änderungen“, die Verbesserungen im Hinblick auf die in der Li-

36 Vgl. Eitel 2003: 43, 47; Dauner-Lieb 2000: 19; Martiny 2002: A 65 ff.; Grziwotz 2001: 66 f.; Schmidt 2007.

37 Röthel 2008; Hüttemann/Rawert 2007; Breitschmid/Künzle 2005: 7. Vgl. aber BGH v. 10. 12. 2003, IV ZR 249/02, NJW 2004: $1382 \mathrm{ff}$.

38 Im österreichischen Recht kann der Pflichtteil vom Erblasser dann auf die Hälfte herabgesetzt werden, wenn er zu seinem Kind „zu keiner Zeit in einem Naheverhältnis [stand], wie es in der Familie zwischen Eltern und Kindern gewöhnlich besteht“, wobei die grundlose Ablehnung des persönlichen Verkehrs durch den Erblasser das Recht auf Pflichtteilsminderung untergehen lässt ( $\$ 773$ a ABGB).

39 Dauner-Lieb 2001: 16, 18 f.; Leisner 2001; Edenfeld 2001: 460. Ablehnend z. B. Röthel in: Lipp/Röthel/Windel 2008: 117; Breitschmid/Künzle 2005: 7.

40 BVerfGE 112, 332.

41 Vgl. Karpen 2007.

42 Vgl. Gaier 2006: 8.

43 Gesetz zur Änderung des Erb- und Verjährungsrechts, vgl. BT-Drs. 96/08; BT-Drs. 16/8954; BT-Drs. 16/13543. Zum Entwurf der Bundesregierung vgl. Meyer 2008; Diwell 2007, Martiny 2007. Die Reform ist am 1.1.2010 in Kraft getreten, vgl. BGBl I $2009,3142 \mathrm{ff}$ 
teratur geäußerte Kritik bringen sollen. Zum einen werden Erleichterungen für die Nachfolgeregelung in Familienunternehmen eingeführt. ${ }^{44}$ Zum anderen werden im Hinblick auf die „Akzeptanz nicht traditionell vorgegebener Lebensentwürfe“45 die Pflichtteilsentziehungsgründe „modernisiert“: Hier wird insofern neuen Familienformen Rechnung getragen, als nicht mehr nur ein Fehlverhalten („nach dem Leben trachten“, schwere Straftaten) gegenüber dem Erblasser selbst, seinem Ehegatten und seinen Nachkommen zu einer Pflichtteilsentziehung berechtigt, sondern dass neu auch Angriffe gegen nichteheliche Lebenspartner und Stiefkinder erfasst sind. ${ }^{46}$ Von einer umfassenden Anpassung des Erbrechts an veränderte Familienrealitäten, etwa im Sinne der oben diskutierten Erweiterung des Kreises der gesetzlichen Erben auf nichteheliche Lebenspartner und Stiefkinder oder durch Lockerung des Pflichtteilsrechts bei nicht gelebter Beziehung kann also nicht die Rede sein.

\section{Bedingungen und Grenzen der Rezeption sozialwissenschaft- lichen Wissens in der erbrechtlichen Reformdebatte}

Welche Chancen hat nun sozialwissenschaftliches Wissen, innerhalb der eben beschriebenen Debatten um die Reform des Erbrechts rezipiert zu werden? Zur Klärung dieser Frage sollen zwei Elemente des erbrechtlichen Diskurses genauer unter die Lupe genommen werden, die eine Art Scharnierfunktion zwischen Rechtswissenschaft und Sozialwissenschaft einnehmen: Es ist dies zum einen die Vorstellung, dass das gesetzliche Erbrecht sich an den familiären „Normalverhältnissen“ und am „durchschnittlichen Erblasserwillen“ orientieren soll. Zum anderen ist es der Begriff der „intergenerationellen Solidarität", der sowohl als normatives Prinzip wie auch als Fragestellung für die sozialwissenschaftliche Erforschung der Rechtswirklichkeit verstanden wird und insbesondere bei der Diskussion um die Legitimation des Pflichtteilsrechts zum dominanten Topos geworden ist.

44 Die Möglichkeiten der Stundung von Pflichtteilsansprüchen werden verbessert $(\mathbb{2} 2331 \mathrm{a}$ Abs. $1 \mathrm{BGB}$ in der neuen Fassung) und die bisherige Zehnjahresfrist für die Berücksichtigung von lebzeitigen Schenkungen an Dritte bei der Berechnung des Pflichtteils wird flexibilisiert (\$2 2325 Abs. 3 BGB in der neuen Fassung). Vgl. dazu Meyer 2008. Die Praxis beurteilt die praktischen Auswirkungen allerdings als eher gering, vgl. nur Reich 2008, Keim 2008.

45 Vgl. BT-Drs. 16/8954: 1.

$46 \sqrt{ } 2333$ Abs. 1 Nr. 1 und 2 BGB in der neuen Fassung. 


\section{Familiäre „Normalverhältnisse“ und die Figur des „durchschnittlichen Erblassers"}

Wenn es um die Ausgestaltung des gesetzlichen Erbrechts geht, ist die rechtswissenschaftliche Reformdebatte geleitet von der Idee, dass es erbrechtliche „Normalverhältnisse“ gibt, an denen sich die Gesetzgebung zu orientieren habe. ${ }^{47}$ Erst wenn die „Normalverhältnisse“ sich verändern - so die rechtswissenschaftliche Perspektive - muss sich auch das Recht anpassen. Der Idee der "Normalverhältnisse“ liegt ein bestimmtes Familienbild zugrunde: Es besteht die Vorstellung, dass sich aus einer bestimmten familiären Bindung, z. B. aus dem Kindesverhältnis, typische erbrechtliche Interessenlagen ergeben, die wiederum in der gesetzlichen Erbfolge ihre Entsprechung finden sollten, z. B. der gleichmäßigen Begünstigung aller Kinder. Neuerdings hat zwar eine Öffnung stattgefunden, indem wie erwähnt diskutiert wird, ob neben den Statusbeziehungen wie Ehe und rechtliches Kindesverhältnis auch Familienbeziehungen berücksichtigt werden sollen, die reine „Realbeziehungen“ sind (soziale Elternschaft, nichteheliche Lebensgemeinschaft). ${ }^{48}$ Der Begriff der „Normalverhältnisse“ wird also - jedenfalls in der Diskussion um mögliches künftiges Recht - nicht mehr notwendigerweise mit der Norm der ehelichen Familie in eins gesetzt. Festgehalten wird aber an der Idee der Homogenität der Lebens- und Interessenlagen innerhalb einer über die äußerliche Form der Beziehung definierten Gruppe, ${ }^{49}$ also beispielsweise der Vorstellung, dass Ehegatten sich typischerweise gegenseitig erbrechtlich begünstigen wollen, oder dass nichteheliche Lebenspartner typischerweise eine solche Begünstigung gerade nicht wollen. ${ }^{50}$

Hauptakteur auf der Bühne der „Normalverhältnisse“ ist die Figur des „durchschnittlichen Erblassers“, dessen „mutmaßlicher Wille“ im Erbrecht seinen Ausdruck finden sollte. ${ }^{51}$ Annahmen über den durchschnittlichen Erblasserwillen beruhen jedoch nicht etwa auf empirischen Erkenntnissen, wie das Adjektiv „durchschnittlich“ nahe legen würde. Die Rechtswissenschaftlerin Anne Röthel spricht deshalb von einem „normativierten“ oder „normativ geläuterten“ Erblasserwillen. ${ }^{52}$ Doch bereits in der Konzentration auf die Position des Erblassers liegt eine Verengung der normativen Perspek-

47 Vgl. etwa BaslerKomm/Breitschmid, Vorbemerkung Art. 467-536 ZGB, N 12.

48 Vgl. oben II. 2.

49 Zur Tendenz zur Homogenisierung von Lebensrealitäten vgl. auch den Beitrag von Baer in diesem Band.

50 Vgl. oben II. 2.

51 Vgl. Fankhauser 2007: 248f. m. w. Nachw.; Kritisch etwa Leipold 1980: 196.

52 Röthel in Lipp/Röthel/Windel 2008: 99. 
tive auf einen einzigen Interessenträger, den Erblasser. Darin spiegelt sich der Erfahrungshorizont der in die erbrechtliche Beratungspraxis involvierten Professionen wider. Dies lässt sich auch in Bezug auf die Reformdebatte zum Pflichtteilsrecht beobachten: Einige juristische Stimmen berufen sich auf die „notarielle Praxis“ und nehmen dabei auf die Interessen ihrer Klienten Bezug. So werden durch das Pflichtteilsrecht verursachte Probleme bei der Regelung von Unternehmensnachfolgen beschrieben, wie auch der Wunsch von Ratsuchenden, besonders enge Beziehungen (und umgekehrt „Entfremdung“) zu einzelnen Kindern, geleistete Pflegedienste, Bedürftigkeit oder wirtschaftlich unverantwortliches Verhalten der Kinder bei der Verfügung über ihren Nachlass berücksichtigen zu können, ohne an den Pflichtteil gebunden zu sein. ${ }^{53}$ Bemängelt wird, dass im Rahmen des geltenden Rechts die Vorstellungen der Erblasser nicht realisiert werden können, „obwohl sie für ihre Wünsche gute, objektiv nachvollziehbare, ja sogar anerkennenswerte Gründe haben. “54 Durch die Verengung auf die Perspektive der nachlassplanenden Klientschaft wird stillschweigend die normative Grundentscheidung getroffen, auf eine Interessenabwägung zwischen den verschiedenen Beteiligten zu verzichten, und stattdessen im Sinne der Privilegierung von Privatautonomie und Testierfreiheit einzig die Interessen der Erblasser zum Maßstab zu nehmen. Von kritischen Stimmen wird denn auch die Selektivität des notariellen Wissens herausgestrichen: Es repräsentiere vor allem die Sicht der Testierenden, wobei insbesondere die Perspektive erbberechtigter Kinder, deren Väter neue Verbindungen eingegangen sind und „welche nunmehr bei der Nachlassplanung stören“, unberücksichtigt zu bleiben drohten. ${ }^{55}$

Der Orientierung am „mutmaßlichen Erblasserwillen“ wird von Kritikern das Prinzip der „Familienbindung“ entgegengesetzt. Das Familienerbrecht finde „seine Rechtfertigung unmittelbar in den ehelichen und familiären Gegebenheiten (tatsächlicher, sittlicher und rechtlicher Art) [...], nicht erst in einem vermuteten Willen des Erblassers, sein Vermögen dem Ehegatten und den nächsten Verwandten zu hinterlassen. “56 Zwar fällt durch diese alternative Orientierung die einseitige Verengung der Perspektive auf eine einzige

53 Vgl. besonders dezidiert Dauner-Lieb 2001.

54 Dauner-Lieb 2001: 462.

55 Martiny 2002: A 69. Diese beschränkte Reichweite ihres Praxiswissens wird zwar von den Notaren selbst zugestanden und es wird als dem empirischen, in sozialwissenschaftlichen Studien erhobenen Wissen unterlegen dargestellt. Gleichzeitig wird aber das Fehlen solcher Studien konstatiert, womit das eigene Erfahrungswissen als zwar zweitklassige aber doch einzig verfügbare Quelle der Erkenntnis erscheint. Vgl. etwa Bengel 2002: L 51.

56 MünchKommBGB/Leipold, 4. Aufl. 2004, Einleitung vor $\mathbb{S} 1922$, Rn. 12. 
Interessensposition weg, eine homogenisierende Sichtweise auf Familie ist aber auch dieser Position eigen.

\section{Der Topos der „intergenerationellen Solidarität“}

Das normative Prinzip der „intergenerationellen“ oder „familiären Solidarität“ ist zum dominanten Topos im Rahmen der Debatte um die Reform des Erbrechts geworden. ${ }^{57}$ Unter dem Titel der „Solidarität“ wird über die Legitimation des Pflichtteilsrechts wie auch von weiteren Regulierungen intergenerationeller Vermögenstransfers wie namentlich im Bereich des Verwandtenunterhaltsrechts diskutiert. ${ }^{58}$ Rechtspolitisch geht es dabei insbesondere um das Verhältnis zwischen öffentlich und privat, zwischen sozialstaatlichen und privaten Leistungen. ${ }^{59}$

Zum einen wird darüber debattiert, ob in Familien heute "Solidarität" überhaupt noch eine handlungsleitende Norm darstelle. Programmatisch ist die Aussage von Helmut Coing anlässlich des 49. Deutschen Juristentags 1972: „Eine Reform des gesetzlichen Erbrechts und des Pflichtteilsrechts sollte nicht ohne sorgfältige empirische Untersuchungen vorgenommen werden. Gegenstand der Untersuchungen sollten nicht nur die in der Bevölkerung herrschenden Auffassungen über die Gestaltung des Erbrechts, sondern auch die tatsächlichen Testiergewohnheiten und vor allem die Frage sein, wieweit in der Familie noch ein tatsächlicher Zusammenhalt herrscht, insbesondere inwieweit über den engsten Familienkreis (Eltern und Kinder) hinaus, die Familie noch eine „Solidaritätsgruppe“ bildet, die sich gegenseitig hilft.“60

Die Sozialwissenschaften selbst stellen denn auch eine verbreitete Praxis des freiwilligen intergenerationellen Austauschs von materiellen und instrumentellen Unterstützungen fest, wobei die Meinungen auseinander gehen, ob die praktizierten Transfers „viel oder wenig“ seien. ${ }^{61}$ In der rechtswissenschaftlichen Rezeption wird aus dieser Feststellung gefolgert, dass „unsere Vermutung, dass die Familiensolidarität gegenüber der Elterngeneration weitgehend nicht mehr gelebt werde, sich in der Forschung nicht bestätigt hat." 62 Von den (vermeintlich eindeutigen) faktischen Feststellungen wird meist ohne weiteres direkt auf die normative Ebene geschlossen: Wenn Soli-

57 Vgl. nur Martiny 2002; Lipp/Röthel/Windel 2008; Zacher 2007; Beckert 2007.

58 Vgl. Martiny 2002.

59 Vgl. Redebeitrag Brudermüller, Ständige Deputation des Deutschen Juristentages 2002: L 141.

6o Coing 1972: A 72.

61 Lüscher 2002: L $24 \mathrm{ff}$.

62 Vgl. Redebeiträge Martiny und Willutzki, Ständige Deputation des Deutschen Juristentages 2002: L 126, L 153. 
darität in den Generationenbeziehungen nach wie vor eine relativ verbreitete Realität ist, dann soll sie auch mittels Rechtspflichten durchgesetzt werden können. Es wird dabei auf die Steuerungsfähigkeit von Recht vertraut: So wird etwa eine negative "Signalwirkung“ an Familien befürchtet, sollte die gegenseitige Unterstützungspflicht von Eltern und Kindern („Verwandtenunterhalt“) abgeschafft werden. ${ }^{63}$ Auch dieser direkte Schluss vom Sein zum Sollen ${ }^{64}$ beruht letztlich auf einer homogenisierenden Sichtweise von Familie, welche die von der Soziologie beschriebene Vielfalt der gelebten Generationenbeziehungen unsichtbar macht.

Eine diametral entgegengesetzte Position, die von der Feststellung einer heterogenen Vielfalt in den aktuellen Generationenbeziehungen ausgeht, sieht die einzige Legitimation für die private Vermögensvererbung in der tatsächlich gelebten Solidarität. So vertritt etwa der an der innerjuristischen Debatte teilnehmende Soziologe Jens Beckert die Auffassung, dass das Pflichtteilsrecht ganz abgeschafft werden müsse, da fixe Pflichtteile für Kinder, Ehepartner oder Eltern gerade in der Zeit der Pluralisierung der Familienformen oftmals nicht mehr der faktischen Nähe und den subjektiven Verpflichtungen entsprächen. ${ }^{65}$ Das rechtspolitische Postulat der Abschaffung des Pflichtteilsrechts wird dabei mit der Auffanglösung der erbrechtlichen Absicherung von bedürftigen oder unterhaltsabhängigen Familienangehörigen verbunden. ${ }^{66}$

\section{Zwischenfazit}

Zusammenfassend lässt sich festhalten, dass in Kontrast zur soziologischen These der Pluralisierung der Familienformen die Rechtswissenschaft - mit wenigen Ausnahmen - an der Idee von typischen Interessenlagen in sauber nach Art der Beziehung (Ehe, Partnerschaft, rechtliches Kindesverhältnis, Stiefkindverhältnis, soziale Elternschaft) abgrenzbaren Konstellationen festhält. Dass dies zu kurz greift, wird deutlich, wenn im Folgenden Resultate jüngerer familiensoziologischer Studien zum Erben in „neuen Familienformen“ herangezogen werden.

63 Vgl. Redebeitrag Brudermüller, Ständige Deputation des Deutschen Juristentages 2002: L 140.

64 Zur Problematik des Übergangs vom Sein zum Sollen vgl. Röhl 1987: 93.

65 Beckert 2007: 9. In eine ähnliche Richtung zielt die von der Rechtswissenschaftlerin Ingeborg Schwenzer im Bereich des Verwandtenunterhaltsrechts vertretene Position, dass mit der Normierung von Rechtspflichten keine Solidarität geschaffen werden könne, wo faktisch keine Solidarität vorhanden sei, vgl. Redebeitrag Schwenzer, Ständige Deputation des Deutschen Juristentages 2002: L 140.

66 Beckert 2007: 17 ff.; so auch Dauner-Lieb 2000: 22 ff. 


\section{Resultate empirischer Untersuchungen zum Erben in „, neuen Familienformen"}

Zwei neuere empirische Studien haben das Erben in „neuen Familienformen" in Deutschland und der Schweiz untersucht. In Deutschland sind die Ergebnisse des von Frank Lettke geleiteten „Konstanzer Erbschaftssurveys“ hervorzuheben, einer Telefonbefragung von Deutschen über 40 Jahren, die im Jahr 2003 durchgeführt wurde. ${ }^{67}$ Für die Schweiz ist die Studie des Büros BASS zu nennen, die unter anderem in einer Telefonbefragung von Schweizer Stimmberechtigten im Jahr 2004 bestand..$^{68}$ In beiden Studien wurden die Einstellungen zur Erbberechtigung verschiedener Personengruppen und das Wissen über die geltende gesetzliche Regelung erfragt. Der Konstanzer Erbschaftssurvey hat zudem das eigentliche Vererbungsverhalten in komplexen Familienformen erforscht.

Auf der Ebene der Einstellungen ergibt sich, dass eine Mehrheit der Befragten, unabhängig von ihrer eigenen Lebenssituation, die Gleichstellung von nichtehelichen Lebenspartnern mit Ehepartnern befürworten: Der Konstanzer Erbschaftssurvey stellt fest, dass 68 Prozent der Personen, die in einer Kernfamilie ${ }^{69}$ leben, eine Erbschaft dann gerecht finden, wenn Lebenspartner so viel erben, als wenn sie Ehepartner wären. Bei den Stieffamilien, ${ }^{70}$ den Patchwork-Familien ${ }^{71}$ und den Kinderlosen sind es sogar zwischen 72 und 81 Prozent, die eine Gleichstellung von Ehe und Lebenspartnerschaft befürworten. ${ }^{72}$ Dieser Befund wird durch die Schweizer Studie bestätigt: Der Aussage „Es sollte beim Erben egal sein, ob man mit dem langjährigen Lebenspartner verheiratet gewesen ist oder nicht" stimmten 75 Prozent der Befragten (eher) zu. ${ }^{73} \mathrm{Im}$ Hinblick auf die erbrechtliche Stellung von eigenen, „leiblichen“ Kindern ${ }^{74}$ im Vergleich zu Stiefkindern, geben fast zwei Drittel

67 Lettke 2007: 107. Vgl. auch Lettke 2006; Lettke 2005; Lettke 2004.

68 Die Befragung wurde im Rahmen der regelmäßigen Univox-Befragungen des GfSForschungsinstituts durchgeführt, Stutz/Bauer/Schmugge 2007: 77. Die Studie hat darüber hinaus anhand einer kantonalen Datenbank den Umfang und die Verteilung von vererbten Vermögen ermittelt; vgl. dazu Breitschmid 2007b.

69 In der Terminologie des KES: verheiratete oder zusammenlebende Eltern mit ausschließlich gemeinsamen Kindern, vgl. Lettke 2006: 3833.

70 Partnerschaften, in denen entweder der Befragte oder dessen Partner nicht gemeinsame Kinder haben.

71 Die Partner haben entweder gemeinsame und nicht gemeinsame Kinder oder beide Partner haben jeweils nicht gemeinsame Kinder.

72 Stieffamilien: $76 \%$, Patchworkfamilien: $72 \%$, Kinderlose: $81 \%$ (Lettke 2006: 3837).

73 Stutz/Bauer/Schmugge 2007: 215.

74 Der Konstanzer Erbschaftssurvey verkennt hier, dass die rechtliche Zuordnung eines Kindes zu einem Vater nicht notwendigerweise mit der biologischen Abstammung 
der Befragten in allen Familienformen an, dass die beiden gleich viel erben sollten. ${ }^{75}$

In Bezug auf das Wissen über die Regelungen des geltenden Erbrechts ist zunächst erstaunlich, wie verbreitet die Falschinformation über die Erbenstellung des unverheirateten Lebenspartners ist: So wissen nur 59 Prozent der im Rahmen des Konstanzer Erbschaftssurveys Befragten, dass Lebenspartner nicht zu den gesetzlichen Erben gehören. ${ }^{76}$ In der Schweizer Studie verfügten sogar nur 52 Prozent der Befragten über dieses Wissen. ${ }^{77}$ Die oben erwähnte Annahme der Rechtswissenschaft, dass die Lebensform der nichtehelichen Lebensgemeinschaft gerade wegen des Verzichts auf rechtliche Absicherung gewählt wird, ${ }^{78}$ erweist sich vor diesem Hintergrund als wenig plausibel. In Bezug auf Stiefkinder sind die Kenntnisse sogar noch schlechter: So sind nur 34 Prozent der in der deutschen Studie Befragten darüber informiert, dass Stiefkinder keine gesetzliche Erbberechtigung haben, ${ }^{79}$ in der Schweizer Studie sind es 37 Prozent. $^{80}$

In Bezug auf das tatsächliche Vererbungsverhalten in den „neuen“, komplexen Familienformen hat der Konstanzer Erbschaftssurvey ergeben, dass in den Stief- und Patchwork-Familien die Absicht, den Partner erbrechtlich $\mathrm{zu}$ begünstigen, signifikant seltener auftritt als bei Kernfamilien und Kinderlosen. Bei den Stiefkindern zeigt sich zudem, dass deutlich weniger als die Hälfte zu den Erben gehören werden, während gemeinsame Kinder fast durchweg begünstigt werden. ${ }^{81}$ Lettke folgert daraus, dass die im Rahmen der Einstellungen festgestellte Norm der Gleichbehandlung aller Kinder, ob gemeinsam oder nichtgemeinsam, zwar auf der diskursiven Ebene vorgebracht, im tatsächlichen Vererbungsverhalten jedoch nicht konsequent umgesetzt wird. ${ }^{82}$

Die Feststellung, dass in Stief- und Patchwork-Familien die Partnerorientierung im Vergleich zu „traditionellen Familien“ eher tiefer ist, wird bestätigt durch eine in England durchgeführte qualitative Studie, die unter anderem die Erbplanung in Patchwork-Familien erfragt hat. So besteht laut der Studie oftmals die Vorstellung, dass in einer zweiten Ehe oder Partnerschaft

übereinstimmen muss.

75 Kernfamilie: $57 \%$, Stieffamilien: $65 \%$, Patchworkfamilien: $62 \%$, Kinderlose: $59 \%$ (Lettke 2006: 3837).

76 Lettke 2004: 288.

77 Stutz/Bauer/Schmugge 2007: 223.

78 Röthel in Lipp/Röthel/Windel 2008, oben II. 2 .

79 Lettke 2004: 288.

8o Stutz/Bauer/Schmugge 2007: 223.

81 Lettke 2006: 3838.

82 Ebenda. 
die Vermögen möglichst getrennt gehalten werden sollten, insbesondere um sicherzustellen, dass im Erbfall die Kinder aus der früheren Beziehung auch wirklich in den Genuss des von ihren (mittlerweile getrennt lebenden) Eltern erwirtschafteten Vermögens gelangen. Es soll quasi kein Vermögen „aus der Familie herausfließen". ${ }^{83}$ Auch zu den Motiven für die nicht durchgängige erbrechtliche Berücksichtigung von Stiefkindern trotz Egalitätsnorm liefert die englische Studie mögliche Anhaltspunkte. Die Autorinnen stellen fest, dass es keine einheitliche Art und Weise gibt, wie neu zusammengesetzte Familien die erbrechtliche Stellung von Stiefkindern behandeln. Entscheidend scheine die Frage der faktischen Verantwortungsübernahme zu sein: Stiefkinder werden eher erbrechtlich begünstigt, wenn der Stiefelternteil sich aktiv an der Erziehung des Kindes seines Partners beteiligt hat. ${ }^{84}$

Die englische Studie macht auch deutlich, dass die Ungleichbehandlung verschiedener Kinder im Rahmen der individuellen Nachlassregelung nicht notgedrungen einen Verstoß gegen die Gleichheitsnorm bedeuten muss. Die Interviews mit geschiedenen und wieder verheirateten Personen fördern eine Unterscheidung in „equality of treatment“ (Gleichbehandlung) und „equality of outcome“ (Ergebnisgleichheit) zu Tage. Demnach beziehen Eltern in Patchwork-Familien oftmals die gesamte Situation ihrer Kinder in die Erbplanung mit ein: So wird bei eigenen Kindern, die aus einer früheren Beziehung stammen auch die voraussichtliche Begünstigung durch den anderen Elternteil (den Ex-Partner) berücksichtigt und mit der Situation von eigenen und Stiefkindern aus der neuen Beziehung verglichen. Dies hat unter Umständen zur Folge, dass in der internen Betrachtung der Patchwork-Familie nicht alle Kinder gleich behandelt werden, um in der Gesamtbetrachtung ein gleiches Ergebnis für alle Kinder zu erreichen. ${ }^{85}$

\section{Perspektiven einer interdisziplinär abgestützten Erbrechtsreform}

In der Perspektive gilt es, den im Rahmen sozialwissenschaftlicher Forschung erzielbaren Erkenntnisgewinn für die Entwicklung eines an veränderte Familienrealitäten angepassten Erbrechts im Rahmen einer interdisziplinär abgestützten Reformdebatte zu nutzen. Interdisziplinarität gilt heute gemeinhin als Ansatz, der die in Disziplinen fragmentierte Wissensproduktion ersetzt durch eine an den zu lösenden Problemen orientierte Herangehensweise, die

83 Finch/Mason 2000: $35 \mathrm{ff}$.

84 Finch/Mason 2000: $47 \mathrm{ff}$.

85 Finch/Mason 2000: 45. 
unterschiedliche disziplinäre Perspektiven im Hinblick auf innovative Lösungsansätze zusammenbringt. ${ }^{86}$ Im Folgenden soll ein pragmatischer Begriff der Interdisziplinarität verwendet werden, der Rechtswissenschaft und Sozialwissenschaft in ihrer je eigenen disziplinären Eigenheit nicht auflösen will, sondern die unterschiedliche erkenntnistheoretische Orientierung für den Erkenntnisprozess nutzen will. ${ }^{87}$ Vor diesem Hintergrund stellt sich die Frage, was der spezifische Beitrag der beiden beteiligten Disziplinen sein könnte.

Die empirischen Erkenntnisse, die die Sozialwissenschaften liefern können, betreffen einerseits das Erfahrungswissen zur Nachlassplanung, über das Menschen verfügen, die in komplexen Familienverhältnissen leben und andererseits das Gebrauchswissen der Rechtspraktikerinnen und -praktiker. $^{88}$

Qualitative sozialwissenschaftliche Untersuchungen können Erkenntnisse zu den normativen Prinzipien liefern, die typischerweise die Nachlassplanung in neu zusammengesetzten Familien orientieren. Da diese nicht in einem normativen Vakuum erfolgt, sondern von den rechtlichen Rahmenbedingungen beeinflusst wird, gilt es, das Zusammenspiel von Normen verschiedener Herkunft zu untersuchen: Untersuchungsgegenstand ist der Normenkomplex im Sinn von Eugen Ehrlichs „lebendem Recht“"89, der in der Begegnung von sozialen Normen, die verwandtschaftliche Beziehungen regulieren (Ehrlichs „gesellschaftliches Recht“) mit notarieller Beratungspraxis (Ehrlichs „Juristenrecht“) und Gesetzesrecht (Ehrlichs „staatliches Recht“) entsteht. ${ }^{90}$ Beispiel eines möglichen Ergebnisses ist die Erkenntnis eines differenzierten Gleichheitsbegriffs, wie sie die oben erwähnte englische Studie zu Tage gefördert hat. Im Sinne der oben festgestellten Mängel einer einzig auf die Interessen der Erblasserin fokussierten Sichtweise wäre dabei die Perspektive aller Familienmitglieder zu erfragen. In Bezug auf den verwendeten Familienbegriff ist wiederum die englische Studie wegweisend: Während sowohl die rechtswissenschaftliche Diskussion wie auch der Konstanzer Erbschaftssurvey von einer Momentaufnahme der Patchwork-Familie ausgeht, die einzig auf die Beziehungen innerhalb der neu zusammengesetzten Familie fokussiert, werden dort auch die Verknüpfungen zu früheren Partnerinnern und Partnern

86 Vgl. etwa Mittelstraß 1996: 11.

87 Weingart 1995: 12. Vgl. auch den Beitrag von Estermann in diesem Band.

88 Zur Terminologie betreffend empirische Erscheinungsformen des Wissens vgl. den Beitrag von Lucke in diesem Band.

89 Ehrlich 1989.

90 „Das lebende Recht ist [ein] gesellschaftliches Recht auf höherer, nämlich auf einer durch Juristenrecht und staatliches Recht beeinflussten Stufe“, Rehbinder 1986: 74. 
und die Verbindungen zwischen nicht zusammenlebenden (Halb-) Geschwistern mit einbezogen. ${ }^{91}$

Untersuchungen, die wohl am ehesten als „rechtstatsächlich“ zu bezeichnen sind, können sodann die Beratungspraxis der Notarinnen und Anwälte erforschen und ermitteln, was typische Regelungen in Testamenten und Erbverträgen bei komplexen Familienverhältnissen sind, wie die Fachleute die gesetzlichen Spielräume und Instrumente für die Interessen ihrer Klientinnen und Klienten nutzen, aber auch auf welche rechtlichen Grenzen sie stoßen. ${ }^{92}$

Die Rechtswissenschaft als soziologische Jurisprudenz muss ihrerseits das konzeptuelle Instrumentarium bereitstellen, um die sozialwissenschaftlichen Erkenntnisse aufnehmen zu können. Wenn wir das Beispiel des gesetzlichen Erbrechts herausgreifen, dann erscheint die herkömmliche rechtswissenschaftliche Konzeption der "Normalverhältnisse“ wenig geeignet für die Rezeption differenzierterer Ergebnisse von qualitativen Studien zum Erben in Stief- und Patchwork-Familien. Die Problematik liegt wie erwähnt in der schematischen und homogenisierenden Konzeption von Familie. Es gilt zum einen die bisher getroffenen statusbasierten Anknüpfungen wie insbesondere die Ehe und das rechtliche Kindesverhältnis zu hinterfragen. Wie die oben wiedergegebenen Untersuchungsergebnisse deutlich machen, hat der Status gerade in neu zusammengesetzten Familien nicht immer die gleiche Bedeutung wie in „traditionellen Kernfamilien“. So wird etwa die zweite Ehepartnerin nicht mehr notwendigerweise als Begünstigte genannt, und Statusbeziehungen zu Kindern verlieren durch den Verlust des Kontaktes zwischen Eltern und Kind an Bedeutung. Auch der Begriff der „Realbeziehung“ erscheint als zu kurz gegriffen, tut sich doch aus den in England geführten Interviews eine Taxonomie auf, die die Kategorien „soziale Elternschaft“ und „faktische Partnerschaft" nochmals in nach der Beziehungsnähe definierte Unterkategorien auffächert. Angesagt ist vor diesem Hintergrund zunächst eine Öffnung der Rechtswissenschaft gegenüber den Kategorisierungen und Prinzipien, die sich aus den Erkenntnissen zu den normativen Orientierungen in Patchwork-Familien ergeben. Das „lebende Recht“ in neu zusammengesetzten Familien erscheint so als Quelle für die Neuentwicklung rechtlicher Konzepte, die nicht an den normativen Erwartungen an Familien orientiert sind, sondern an der gelebten Beziehungspraxis.

91 Vgl. auch Strathern 2005: 27.

92 Bereits die heute als Pionierleistung der Rechtstatsachenforschung bezeichnete Umfrage von Martin Wolff von 1906 erfragte unter anderem die notarielle Praxis im Bereich des Erbrechts; vgl. Heldrich 1986. 
Ganz grundsätzlich fragt sich dabei, ob das Recht angesichts einer heterogenen Vielfalt von Familienkonstellationen, Beziehungsintensitäten und Individualinteressen noch an einer Regelungstechnik festhalten will, die die soziale Wirklichkeit in fixen rechtlichen Kategorien abbilden will. Eine Alternative könnte in der Verschiebung des Fokus auf Prozess und Vertrag liegen. ${ }^{93}$ An die Stelle eines auf den Erblasser, seinen „mutmaßlichen Willen“ und seine Privatautonomie zentrierten Rechts, würde ein Recht treten, das an den Zielen der nach einem Todesfall anstehenden Reorganisation von (Familien-) Beziehungen und des Interessensausgleichs orientiert ist. Dies würde nicht zuletzt den historischen Entwicklungen des familienbezogenen Rechts von Status zu Vertrag und Beziehung ${ }^{94}$ und von der alleinigen Entscheidungsgewalt des pater familias hin zur gleichberechtigten Mitsprache aller Familienmitglieder entsprechen. Der rechtswissenschaftliche Beitrag zur Erbrechtsreform würde in diesem Rahmen in der Entwicklung rechtlicher Instrumente liegen, die auf Prozeduralisierung und Kontraktualisierung hinwirken, etwa durch Schaffung von Anreizen zur vertraglichen Regelung des intergenerationellen Vermögenstransfers oder durch Einrichtung von behördlich unterstützten Verfahren, die nicht autoritative Entscheidungen über erbrechtliche Positionen, sondern die innerfamiliäre Aushandlung von Vermögensumverteilungen zum Ziel haben.

Von Seiten der Sozial- wie der Rechtswissenschaften ist also einige Vorarbeit und Wissensproduktion notwendig, um die Grundlage für eine auf die veränderten Familienstrukturen reagierende Erbrechtsreform zu schaffen. Wesentlich für den Erfolg dieses Unterfangens ist, dass die beiden Wissenschaftszweige zugleich ihrem eigenen Erkenntnisinteresse treu bleiben und sich gegenüber den Fragestellungen der anderen Disziplin öffnen: Weder eine Kooperation im Sinne des Musters „Jurist(inn)en fragen - Soziolog(inn)en antworten "95 noch eine völlig von rechtspolitischen Fragestellungen abgekoppelte Soziologie des Erbens würden zum Ziel führen.

\section{Literatur}

Bauszus, Silvia (2006) Der Topos von der „Großfamilie“ in der familien- und erbrechtlichen Diskussion, Frankfurt a.M.

93 Für das Familienrecht vgl. grundlegend Büchler 2008 und Cohen 2002.

94 Vgl. Schwenzer 2001: 200.

95 Machura (2001): 295. 
Beckert, Jens (2004) Unverdientes Vermögen. Soziologie des Erbrechts, Frankfurt a.M.

Beckert, Jens (2007) Familiäre Solidarität und die Pluralität moderner Lebensformen - Eine gesellschaftstheoretische Perspektive auf das Pflichtteilsrecht, in: Röthel, Anne (Hrsg.), Reformfragen des Pflichtteilsrechts, Köln: $1 \mathrm{ff}$.

Bengel, Manfred (2002) Referat, in: Ständige Deputation des Deutschen Juristentages (Hrsg.), Verhandlungen des vierundsechzigsten Deutschen Juristentages Bd. II/1, München: L $51 \mathrm{ff}$.

Breitschmid, Peter (2001) Erbrecht. Unter Berücksichtigung insbesondere der Schnittstellen von persönlichkeits- und vermögensrechtlichen Aspekten, in: Gauch, Peter (Hrsg.), Die Rechtsentwicklung an der Schwelle zum 21. Jahrhundert. Standortbestimmung und Ausblick der Herausgeber, Autorinnen und Autoren des Kommentars zum Schweizerischen Zivilgesetzbuch („Zürcher Kommentar"), Zürich: $109 \mathrm{ff.}$

Breitschmid, Peter (2007a) Das Erbrecht des 19. im 21. Jahrhundert. Der Konflikt zwischen Status, Realbeziehung und erblasserischer Freiheit, Successio: $6 \mathrm{ff}$.

Breitschmid, Peter (2007b) „Erben in der Schweiz“. Glück oder Last? Verdient oder unverdient? Successio: $202 \mathrm{ff}$.

Breitschmid, Peter / Künzle, Hans Rainer (2005) „Pflichtteile behindern den Erblasser übermäßig“. Interview, Gesprächsleitung: René Schuhmacher, Daniela Schwegler, plädoyer: $6 \mathrm{ff}$.

BüChler, Andrea (2008) Kulturelle Vielfalt und Familienrecht. Die Bedeutung kultureller Identität für die Ausgestaltung europäischer Familienrechtsordnungen - am Beispiel islamischer Rechtsverständnisse, in: Deutsche Gesellschaft für Völkerrecht (Hrsg.), Pluralistische Gesellschaften und internationales Recht, Heidelberg: $215 \mathrm{ff}$.

Cohen, Jean L. (2002) Regulating intimacy. A new legal paradigm, Princeton NJ.

Coing, Helmut (1972) Empfiehlt es sich, das gesetzliche Erb- und Pflichtteilsrecht neu zu regeln? in: Ständige Deputation des Deutschen Juristentages (Hrsg.), Verhandlungen des neunundvierzigsten Deutschen Juristentages, München: A11ff.

Dauner-Lieb, Barbara (2000) Das Pflichtteilsrecht - Ketzerische Fragen an ein altehrwürdiges Institut, Forum Familien- und Erbrecht: 16 ff.

Dauner-Lieb, Barbara (2001) Bedarf es einer Reform des Pflichtteilsrechts? Deutsche Notar-Zeitschrift: $460 \mathrm{ff}$

Diwell, Lutz (2007) Reformen im Erb- und Pflichtteilsrecht - Spielräume aus Sicht des Bundesministeriums der Justiz, in: Röthel, Anne (Hrsg.), Reformfragen des Pflichtteilsrechts, Köln: 185 ff.

Druey, Jean Nicolas (2002) Grundriss des Erbrechts, Bern.

EDENFELD, STEFAn (2001) Europäische Entwicklungen im Erbrecht, Zeitschrift für Erbrecht und Vermögensnachfolge: $457 \mathrm{ff}$ 
Ehrlich, Eugen (1989) Grundlegung der Soziologie des Rechts, 4. Aufl., Berlin [1913].

Eitel, Paul (2003) KMU und Pflichtteilsrecht, in: Girsberger, Daniel (Hrsg.), Rechtsfragen rund um die KMU, Zürich: $43 \mathrm{ff}$.

Fankhauser, Roland (2007) Gesetzliches Ehegattenerbrecht in der (Ehe-) Krise, Die Praxis des Familienrechts: $229 \mathrm{ff}$.

Finch, Janet / Mason, Jennifer (2000) Passing on. Kinship and inheritance in England, London.

Gaier, Reinhard (2006) Die Bedeutung der Grundrechte für das Erbrecht, Zeitschrift für Erbrecht und Vermögensnachfolge: $2 \mathrm{ff}$.

Grziwotz, Herbert (2001) Rechtspolitische Probleme aus kautelarjuristischer Sicht, in: Henrich, Dieter (Hrsg.), Familienerbrecht und Testierfreiheit im europäischen Vergleich, Bielefeld: $53 \mathrm{ff}$.

Hausheer, Heinz / Aebi-Müller, Regina (2001) Familienerbrecht und Testierfreiheit in der Schweiz, in: Henrich, Dieter (Hrsg.), Familienerbrecht und Testierfreiheit im europäischen Vergleich, Bielefeld: $213 \mathrm{ff}$

Heldrich, Andreas (1986) Die Bedeutung der Rechtssoziologie für das Zivilrecht, Archiv für die civilistische Praxis: $74 \mathrm{ff}$.

Hüttemann, Rainer / Rawert, Peter (2007) Pflichtteil und Gemeinwohl - Privilegien für gute Zwecke? in: Röthel, Anne (Hrsg.), Reformfragen des Pflichtteilsrechts, Köln: $73 \mathrm{ff}$.

Karpen, Ulrich (2007) Das Pflichtteilsrecht im Spannungsfeld von Einzelnem, Familie, Gesellschaft und Staat, in: Röthel, Anne (Hrsg.), Reformfragen des Pflichtteilsrechts, Köln: 169 ff.

Keim, Christopher (2008) Die Reform des Erb- und Verjährungsrechts und ihre Auswirkungen auf die Gestaltungspraxis, Zeitschrift für Erbrecht und Vermögensnachfolge: $161 \mathrm{ff}$.

Kissling, Hans (2008) Reichtum ohne Leistung. Die Feudalisierung der Schweiz, Zürich.

Leipold, Dieter (1980) Wandlungen in den Grundlagen des Erbrechts? Archiv für die civilistische Praxis: $160 \mathrm{ff}$.

Leisner, Walter (2001) Pflichtteilsentziehungsgründe nach $\$ \$ \$ S 333 \mathrm{ff}$. BGB verfassungswidrig? - BVerfG lässt eine wichtige Frage offen, Neue Juristische Wochenschrift: $126 \mathrm{ff}$.

LetTke, Frank (2004) Subjektive Bedeutungen des Erbens und Vererbens. Ergebnisse des Konstanzer Erbschafts-Surveys, Zeitschrift für Soziologie der Erziehung und Sozialisation: $277 \mathrm{ff}$.

LetTKe, FrAnk (2005) Vererbungspläne in unterschiedlichen Familienformen, Schweizerische Zeitschrift für Soziologie: $407 \mathrm{ff}$.

LetTKe, Frank (2006) Vererbungsmuster in unterschiedlichen Familienformen, in: Deutsche Gesellschaft für Soziologie (Hrsg.), Soziale Ungleichheit, kulturelle 
Unterschiede. Verhandlungen des 32. Kongresses der Deutschen Gesellschaft für Soziologie in München 2004, Frankfurt a. M.: 3831 ff.

LetTKe, Frank (2007) Vererbungsabsichten in unterschiedlichen Familienformen. Ein Beitrag zur Institutionalisierung generationaler Kontinuität, in: Lettke, Frank/ Lange, Andreas (Hrsg.), Generationen und Familien. Analysen - Konzepte - gesellschaftliche Spannungsfelder, Frankfurt a. M.: $96 \mathrm{ff}$.

Linker, Anja Celina (1999) Zur Neubestimmung der Ordnungsaufgaben im Erbrecht in rechtsvergleichender Sicht. Eine rechtsvergleichende Untersuchung unter Berücksichtigung des deutschen und französischen Rechts, Tübingen.

Lipp, Volker / Röthel, Anne / Windel, Peter A. (2008) Familienrechtlicher Status und Solidarität, Tübingen.

LuCKe, Doris (1988) Zu diesem Heft: ,Verwendung soziologischen Wissens in juristischen Zusammenhängen', Zeitschrift für Rechtssoziologie: $121 \mathrm{ff}$.

LÜSCHER, KURT (2002) Widersprüchliche Mannigfaltigkeit - Nachhaltige Leistungen: Ehe, Familie und Verwandtschaft heute, in: Ständige Deputation des Deutschen Juristentages (Hrsg.), Verhandlungen des vierundsechzigsten Deutschen Juristentages Bd. II/1, München: L9-L50 ff.

Machura, Stefan (2001) Die Aufgabe(n) der Rechtssoziologie. Eine Antwort an Theo Rasehorn, Zeitschrift für Rechtssoziologie: $293 \mathrm{ff}$.

Martiny, Dieter (2002) Empfiehlt es sich, die rechtliche Ordnung finanzieller Solidarität zwischen Verwandten in den Bereichen des Unterhaltsrechts, des Pflichtteilsrechts, des Sozialhilferechts und des Sozialversicherungsrechts neu zu gestalten? Unterhalts- und erbrechtliches Teilgutachten. Gutachten A für den 64. Deutschen Juristentag (Berlin 2002), in: Ständige Deputation des Deutschen Juristentages (Hrsg.), Verhandlungen des vierundsechzigsten Deutschen Juristentages Bd. I, München: A $11 \mathrm{ff}$.

Martiny, Dieter (2007) Zur Reform des Pflichtteilsrechts, in: Röthel, Anne (Hrsg.), Reformfragen des Pflichtteilsrechts, Köln: 195 ff.

Meyer, Thomas (2008) Erbrechtsreform als Reaktion auf die gesellschaftlichen Veränderungen, Familie Partnerschaft Recht: $537 \mathrm{ff}$.

Mittelstraß, Jürgen (1996) Stichwort Interdisziplinarität, Basel.

Monahan, John / Walker, Laurens (2008) Judicial Use of Social Science Research, in: Mertz, Elizabeth (Hrsg.), The Role of Social Science in Law, Aldershot: $23 \mathrm{ff}$.

Muscheler, Karlheinz (2006) Stieffamilie, Status und Personenstand, Das Standesamt: $189 \mathrm{ff}$.

Otte, Gerhard (2002) Das Pflichtteilsrecht - Verfassungsrechtsprechung und Rechtspolitik, Archiv für die civilistische Praxis: $317 \mathrm{ff}$.

Peuckert, RÜdiger (2008) Familienformen im sozialen Wandel, Wiesbaden.

Rauscher, Thomas (1993) Reformfragen des gesetzlichen Erb- und Pflichtteilsrechts, Regensburg. 
Rehbinder, Manfred (1986) Die Begründung der Rechtssoziologie durch Eugen Ehrlich, Berlin.

Reich, Thomas (2008) Neue Stundungsgründe für Pflichtteilsansprüche, Familie Partnerschaft Recht: $555 \mathrm{ff}$.

Reimann, Wolfgang (2001) Familienerbrecht und Testierfreiheit im deutschen Recht, in: Henrich, Dieter (Hrsg.), Familienerbrecht und Testierfreiheit im europäischen Vergleich, Bielefeld: $33 \mathrm{ff}$.

RÖHL, Klaus Friedrich (1987) Rechtssoziologie. Ein Lehrbuch, Köln.

RöThel, AnNe (2006) Lebensformen - Status - Personenstand: rechtsvergleichend und rechtspolitisch betrachtet, Das Standesamt: $34 \mathrm{ff}$.

Röthel, Anne (Hrsg.) (2007) Reformfragen des Pflichtteilsrechts, Köln.

Röthel, Anne (2008) Was bringt die Pflichtteilsreform für Stiftungen? Zeitschrift für Erbrecht und Vermögensnachfolge: $112 \mathrm{ff}$.

Schaer, Walter (1976) Ist das Pflichtteilsrecht ein erhaltenswertes Institut? Eine rechtspolitische Betrachtung zum schweizerischen Pflichtteilsrecht mit rechtsvergleichenden Hinweisen, Zürich.

SChlüter, Wilfried (2000) Die Änderung der Rolle des Pflichtteilsrechts im sozialen Kontext, in: Canaris, Claus-Wilhelm et al. (Hrsg.), 50 Jahre Bundesgerichtshof. Festgabe aus der Wissenschaft, München: $1047 \mathrm{ff}$.

Schmidt, Karsten (2007) Pflichtteil und Unternehmensnachfolge. Rechtspolitische Überlegungen im Schnittfeld von Erbrecht und Unternehmensrecht, in: Röthel, Anne (Hrsg.), Reformfragen des Pflichtteilsrechts, Köln: $37 \mathrm{ff}$.

Schwenzer, Ingeborg (1987) Vom Status zur Realbeziehung. Familienrecht im Wandel, Baden-Baden.

Schwenzer, Ingeborg (2001) The Evolution of Family Law - From Status to Contract and Relation, European Journal of Law Reform: $199 \mathrm{ff}$.

SchWenzer, Ingeborg (2007) Grundlinien eines modernen Familienrechts aus rechtsvergleichender Sicht, Rabels Zeitschrift für ausländisches und internationales Privatrecht: $705 \mathrm{ff}$.

Seibt, Constantin (2008) „Die Schweiz gleicht einem Feudalstaat“, Tagesanzeiger, 14.5.2008: $13 \mathrm{ff}$.

Ständige Deputation des Deutschen Juristentages (Hrsg.) (2002) Verhandlungen des vierundsechzigsten Deutschen Juristentages Bd. II/2, München (II/2).

Strathern, Marilyn (2005) Kinship, law and the unexpected. Relatives are always a surprise, New York.

Strätz, Hans-Wolfgang (2001) Reform der gesetzlichen Erbfolge, Deutsche NotarZeitschrift: $452 \mathrm{ff}$.

Strebel, Lorenz (2008) Gesetzliches Erbrecht, Pflichtteil und Konkubinat. Thesen zu einer Anpassung des Erbrechts an die Lebensrealität, Aktuelle Juristische Praxis: $1029 \mathrm{ff}$. 
Stutz, Heidi / Bauer, Tobias / Schmugge, Susanne (2007) Erben in der Schweiz. Eine Familiensache mit volkswirtschaftlichen Folgen, Zürich.

Teubner, Gunther (1990) Die Episteme des Rechts. Zu erkenntnistheoretischen Grundlagen des reflexiven Rechts, in: Grimm, Dieter (Hrsg.), Wachsende Staatsaufgaben - sinkende Steuerungsfähigkeit des Rechts, Baden-Baden: $115 \mathrm{ff}$.

Teubner, Gunther (2004) Netzwerk als Vertragsverbund. Virtuelle Unternehmen, Franchising, Just-in-time in sozialwissenschaftlicher und juristischer Sicht, Baden-Baden.

Tyrell, Hartmann (1988) Ehe und Familie - Institutionalisierung und Deinstitutionalisierung, in: Lüscher, Kurt/Schultheis, Franz/Wehrspaun, Michael (Hrsg.), Die „postmoderne“ Familie, Konstanz: $145 \mathrm{ff}$.

Weingart, Peter (1995) Die Einheit der Wissenschaft - Mythos und Wunder, in: Weingart, Peter (Hrsg.), Grenzüberschreitungen in der Wissenschaft, Baden-Baden: $11 \mathrm{ff}$.

Willekens, Harry (2006) The Puzzle of Inheritance Law's Resistance to Social Change, in: Swennen, F./Barbaix, R. (Hrsg.), Over erven. Liber amicorum Mieken Puelinckx-Coenen, Mechelen: $513 \mathrm{ff}$.

Wolf, Stephan (2007) Ist das schweizerische Erbrecht in seinen Grundlagen revisionsbedürftig? Zeitschrift des Bernischen Juristenvereins: $301 \mathrm{ff}$.

Zacher, Hans F. (2007) Pflichtteil und intergenerationelle Solidarität, in: Röthel, Anne (Hrsg.), Reformfragen des Pflichtteilsrechts, Köln: 135 ff. 